\title{
Price Signalling Differentiated Experience Goods: Are Uniform Movie Prices a Puzzle?*
}

\author{
In-Uck Park** $\cdot$ Joon Song***
}

\begin{abstract}
In a simple static model of differentiated experience goods supplied by a single seller, we show that both a uniform price equilibrium and a price signalling equilibrium coexist. This is in contrast to the received wisdom that price signalling of quality is nonviable in static settings. We also show that the seller's profit is always higher in the price signalling equilibrium than in the uniform price equilibrium, but the consumer surplus and social welfare may be higher in either equilibrium depending on the distribution of the consumers' tastes for the differentiated goods.
\end{abstract}

JEL Classification: D40, D82

Keywords: Experience Good, Movie Pricing, Price Signalling, Uniform Pricing

\section{Introduction}

In our living memory, movie theaters have persistently charged identical prices for movies that differ in various dimensions. Although observed across differentiated products of other kinds as well (e.g, downloadable music and tickets to football matches with different teams), uniform pricing across movies is perceived as particularly puzzling given the large variance both in their production costs and anticipated demand/popularity. ${ }^{1}$ On the other hand, differential pricing is observed for various other experience goods, and some studies attribute a significant part of it

Received: Feb. 9, 2020. Revised: July 1, 2020. Accepted: Aug. 27, 2020.

* We thank a Co-Editor and two anonymous referees for constructive comments that improved the paper significantly. This work was supported by the National Research Foundation of Korea Grant funded by the Korean Government (NRF-2019S1A5A2A01047656).

** First Author, Professor, Department of Economics, University of Bristol, Bristol, UK, E-mail: i.park@bristol.ac.uk

** Corresponding Author, Professor, Department of Economics, Sungkyunkwan University, 25-2 Sungkyunkwan-ro, Jongno-gu, Seoul 03063, Korea, E-mail: joonsong@skku.edu

${ }^{1}$ More popular movies are sold more through running longer. But, the standard theory generally predicts a higher price as well as a larger quantity when the demand is bigger. 
to quality differences in some markets, such as wine (Miller, Stone, and Stuen, 2013).

As a signalling device, prices face an inherent issue in that they do not incur different costs across quality levels in ways conducive to separation, thus failing the so-called single-crossing property which is key to effective signalling. If sellers face repeat-purchase customers, however, this issue is alleviated and price signalling is shown to be possible when the seller chooses a price in conjunction with an advertisement level (Nelson, 1970; Milgrom and Roberts, 1986) or customers also possess private, individual-specific information on quality (Judd and Riordan, 1994).

Advertising is indeed prevalent in movie markets. But, effective signalling of quality by advertising is likely to result in differential (rather than uniform) pricing across movies (Milgrom and Roberts, 1986). ${ }^{2}$ In addition, people seldom purchase/watch the same movie repeatedly at the theater. Therefore, the repeat purchase models above do not seem to account for the observed movie prices fully.

Alternatively, Wolinsky (1983) shows that prices may serve as signals when competitive firms produce different quality at differential cost and consumers get imperfect information on quality at a cost sequentially until purchase. ${ }^{3}$ However, most theaters in the US possess some geographic market power because they are the only theater in town or they have exclusive licensing according to Orbach and Einav (2007) who suggest that differential pricing, though dismissed by industry practitioners, could benefit movie exhibitors.

In light of the discussions above, movies may be treated as differentiated experience goods without repeat purchase supplied by a single supplier at the retail level. In such static monopoly markets for experience goods, price signalling of quality is known to be ineffective because the single-crossing property typically fails as mentioned above. If this general insight prevails for movie theaters as well, then differential pricing of movies would not even be feasible, let alone beneficial. Even if price signalling proves feasible, its plausability depends on whether uniform pricing is also viable and how the two pricing regimes compare in various criteria. To assess how puzzling uniform pricing is and what its implications may be, therefore, one needs first to understand possible equilibrium behavior and its welfare properties in such markets.

To this end, we develop a simple model of differentiated experience goods supplied by a single seller, and examine equilibrium pricing behavior and its welfare implications. Specifically, a profit-maximizing seller prices two movies of different qualities in distinct genres, for customers who decide which movie to

\footnotetext{
${ }^{2}$ Also, insofar as movie advertising tends to be uninformative and for the sake of "burning money", the cost is unlikely to depend on the movie quality and the single-crossing property is unlikely to be met.

${ }^{3}$ On the other hand, Heidhues and Koszegi (2008) shows that uniform pricing results from price competition among sellers of differentiated goods to attract loss-averse consumers.
} 
watch (or not at all) based on their private tastes for the two genres as well as the prices. The customers do not observe the qualities of the two movies, but make inferences about them from the prices set by the seller. Their tastes for the two genres are symmetrically distributed.

The first finding is that both the uniform pricing and differential pricing/price signalling are supported as equilibrium depending on whether or not the customers associate high prices with higher quality. Such association is nonviable if the seller supplies a single experience good because she would always claim high quality by pricing high. In contrast, we show that signalling quality is possible when multiple, differentiated experience goods are supplied, because the seller would internalize the strategic externality in a way that the incentive to overprice a low-quality good is mitigated by the incentive to extract a higher surplus from the consumers looking for a high-quality good. ${ }^{4}$ A uniform price equilibrium also exists by the usual logic of coordination failure, namely, that (price) signalling does not work if no one anticipates it. Both the uniform price equilibrium and the price signalling equilibrium are stable in the sense of satisfying the Intuitive Criterion of Cho and Kreps (1987).

We then compare the two equilibria from the perspectives of the seller, the customers and the social welfare. First, we show that the seller's profit is always higher in the price signalling equilibrium than in the uniform price equilibrium. This result stems from the fact that customers make more efficient purchase decisions due to price signalling of quality, hence they are willing to pay more on average.

The next question is whether more efficient choices benefit consumers as well, or they are exploited to boost the seller's profit. If the latter, does the boosted profit overshadow the reduction in consumer surplus so that the total welfare is higher with price signalling? The answers to these questions turn out to depend on the distribution of consumers' tastes/types.

We show, by providing sufficient conditions, that price signalling improves both consumer surplus and social welfare when customer tastes are distributed relatively smoothly, bringing about Pareto-improvement upon uniform pricing. Relative to uniform price equilibrium, the seller boosts sale of high quality at the expense of low quality by reducing quality-adjusted price for the former but raising it for the latter. The rise in the latter price is small when tastes are smoothly distributed because then the revenue loss from raised price looms large given the already reduced volume of low quality sale.

On the flip side, consumer surplus and social welfare may decrease with price signalling if the distribution of tastes is sufficiently volatile: in this case, the rise in low quality price may be drastic as the corresponding loss in sale can be small. We

\footnotetext{
${ }^{4}$ We thank an anonymous referee for suggesting this intuition.
} 
provide a robust example that illuminates the underlying intuition.

We motivated our study with the movie industry, but it sheds new light on the pricing practices and their implications in markets for differentiated experience goods in general. Our results suggest that both uniform pricing and price signalling can prevail in equilibrium, supported by differently coordinated beliefs of consumers on the informational contents of the prices set by the seller. Although the seller always prefers differential pricing as it generates a higher profit, the consumers and the society as a whole may prefer uniform pricing depending on the composition of consumer tastes. Therefore, different pricing practices in different markets are not only consistent with equilibrium but also possibly welfareenhancing. Such divergent welfare comparisons depending on the market characteristics carry potential policy implications as well.

The model in this paper is very stylized in order to focus on the potential of price signalling differentiated experience goods. Yet, we hope that it serves as a base model that may be extended to address interesting related issues such as the effect of competition, role of industry structure, and policy analysis. We leave such extensions as future work.

The paper is organized as follows. Section 2 describes the model. Sections 3 and 4 characterize uniform price equilibrium and price signalling equilibrium, respectively. Section 5 compares the two equilibria in terms of stability, profits and welfare. Section 6 clarifies the extent to which our results apply. Section 7 concludes.

\section{Model}

A movie exhibitor/multiplex, referred to as the seller, releases two movies, $A$ and $B$, in different genres which we also denote as $A$ and $B$ with a slight abuse of notation. The seller observes the quality ${ }^{5}$ of the two movies and sets a price $p_{m} \geq 0$ for each movie $m \in\{A, B\}$ to maximize her expected profit. We assume that the marginal cost is 0 (i.e., the movies are already produced) and there is no capacity constraint.

Each movie $m \in\{A, B\}$ is one of three possible qualities, high $\left(q_{m}=h\right)$ or low $\left(q_{m}=\ell\right.$ ) with equal probability, but sometimes it turns out to be a complete flop, which we describe as "zero" quality $\left(q_{m}=0\right)$, with a small probability $\varepsilon>0$. To facilitate exposition, we present our analysis assuming that either one movie is of high quality and the other is of low quality, or both are of zero quality. ${ }^{6}$ That is,

\footnotetext{
5 The quality can be interpreted as the likelihood that the movie will be received well by the viewers. The exhibitor has an informational advantage in estimating it.

${ }^{6}$ Although realistic, allowing for zero quality is inessential for our results but simplifies exposition as clarified later.
} 
$\left(q_{A}, q_{B}\right)=(h, \ell)$ and $\left(q_{A}, q_{B}\right)=(\ell, h)$ with probability $(1-\varepsilon) / 2$ each and $\left(q_{A}, q_{B}\right)=(0,0)$ with a small probability $\varepsilon>0$. However, we clarify in Section 6 that our main analysis and results hold for more general distributions of movie quality, in particular, when there is a broad range of quality levels, as well as when $q_{A}$ and $q_{B}$ are independent.

A representative consumer (of a continuum of ex-ante identical consumers of measure 1) knows the distribution of the movie quality pair specified above, but does not observe the realized qualities. He has a private taste $t_{m} \in[0,1]$ for the genre $m \in\{A, B\}$ of each movie. His type is $t=\left(t_{A}, t_{B}\right)$ drawn from a joint probability distribution $\mu$ on $T:=[0,1]^{2}$ with a full support, which is symmetric between the two movies:

$$
\mu(X)=\mu\left(\left\{\left(t_{A}, t_{B}\right) \mid\left(t_{B}, t_{A}\right) \in X\right\}\right) \text { for all } X \subset T
$$

From watching a movie $m$ for a price $p_{m}$, the consumer derives a surplus of $q_{m} \cdot t_{m}-p_{m}$ where $q_{m} \in Q:=\{h, \ell, 0\}$ denotes the realized quality and $h$ and $\ell$ are real number such that $0<\ell<h$. The consumer watches at most one movie and maximizes his expected surplus.

A seller's strategy is a mapping $\sigma_{s}$ from each possible quality pair $q=\left(q_{A}, q_{B}\right)$ to a price vector $p=\left(p_{A}, p_{B}\right)$ which may be restricted to $p \in P:=[0, h]^{2}$ because the proceeds from any price exceeding $h$ are zero. Facing a price vector $p$, a consumer of type $t=\left(t_{A}, t_{B}\right)$ maximizes his expected surplus by

$$
\begin{cases}\text { watching } A \text { if } & q_{A}(p) t_{A}-p_{A} \geq \max \left\{q_{B}(p) t_{B}-p_{B}, 0\right\} \\ \text { watching } B \text { if } & q_{B}(p) t_{B}-p_{B} \geq \max \left\{q_{A}(p) t_{A}-p_{A}, 0\right\} \\ \text { not watching if } & 0>\max \left\{q_{A}(p) t_{A}-p_{A}, q_{B}(p) t_{B}-p_{B}\right\}\end{cases}
$$

where $q_{m}(p)=\sum_{q \in Q} q \cdot \beta_{m}(q \mid p) \in[0, h]$ is the expected quality of movie $m$ obtained from the posterior belief $\beta_{m}(\cdot \mid p) \in \Delta(Q)$ on its quality, conditional on the price vector $p$ set by the seller. Thus, a strategy of the consumer is a function

$$
\sigma_{c}: T \times P \rightarrow\{A, B, \emptyset\}
$$

where $\sigma_{c}(t, p) \in\{A, B, \emptyset\}$ encodes watching the movie $A, B$, or neither, contingently on his type and price vector. ${ }^{7}$

A strategy profile $\left(\sigma_{s}, \sigma_{c}\right)$ and a belief profile $\beta=\left(\beta_{A}, \beta_{B}\right): P \rightarrow \Delta(Q) \times \Delta(Q)$ constitute a (perfect Bayesian) equilibrium if $(i) \sigma_{c}$ satisfies (2) given $p$ and $\beta$,

\footnotetext{
${ }^{7}$ Note that we only consider pure strategies. This is innocuous because a mixed strategy is optimal only for a measure zero set of types for every $p$ by (2).
} 
(ii) $\sigma_{s}(q)$ maximizes the seller's expected profit given $\sigma_{c}$ for each possible quality pair $q$, and (iii) $\beta$ conforms to Bayes rule whenever possible.

\section{Uniform Price Equilibrium}

We start with the possibility of uniform pricing, that is, the seller sets the same price for both movies, $p_{u}=p_{A}=p_{B}$, independently of the realized quality. Rather than restricting the seller to set only one price for both, we explain it as equilibrium behavior in our model (where the seller is free to set different prices).

In such equilibrium, the seller opts to set the identical equilibrium prices inducing the consumer to perceive both movies of the same average quality and behave accordingly, because any other prices would provoke the consumer's beliefs and responses that are less lucrative. As such off-equilibrium beliefs, we postulate that the consumer perceives both movies as zero quality if priced differently:

$$
\beta_{A}\left(0 \mid p_{A}, p_{B}\right)=\beta_{B}\left(0 \mid p_{A}, p_{B}\right)=1 \text { if } p_{A} \neq p_{B}
$$

Then, the seller is deterred from pricing the two movies differently because the customer would watch neither movie, believing both are of zero quality.

As is usually the case in signalling games, a plethora of other off-equilibrium beliefs also work so long as they assign a sufficiently low expected quality to the movie priced higher than the equilibrium uniform price. Such beliefs always exist provided that the minimal quality, however unlikely, is low enough, the precise level of which depends on the distribution $\mu .{ }^{8}$ Hence, we adopt a minimal quality of zero which clearly works for all distributions and thus, simplifies exposition. This is the only role played by zero quality in this paper. For notational ease, therefore, in the rest of the paper we present the analysis for the limit case that $\varepsilon=0 .{ }^{9}$

As differential pricing would attract no customer due to (3), the seller sets identical prices $p_{u}$ for both movies. Then, the consumer decides to watch one of them if and only if

$$
\bar{q} \cdot \max \left\{t_{A}, t_{B}\right\} \geq p_{u} \text { where } \bar{q}=\frac{h+\ell}{2}
$$

\footnotetext{
${ }^{8}$ For example, the minimal quality may be $\ell$ if $\mu$ is a uniform distribution.

${ }^{9}$ Keeping $\varepsilon>0$ complicates exposition without changing the results. For instance, the average quality would be $\bar{q}=\frac{h+\ell}{2}(1-\varepsilon)$ instead of $\frac{h+\ell}{2}$ in the next paragraph. In fact, the main analysis remains valid when $h$ ( $\ell$, resp.) is reinterpreted as the expected value when the quality is high (low, resp.) with probability $1-\varepsilon$ and zero with probability $\varepsilon$.
} 
which is the case with probability $\mu\left(T\left(p_{u}\right)\right)$ where $T\left(p_{u}\right)=\left\{t \mid \bar{q} \cdot \max \left\{t_{A}, t_{B}\right\}\right.$ $\left.\geq p_{u}\right\}$ is the set of consumer types whose expected surplus from watching the movie in his favorite genre is positive. Hence, the seller faces the standard profit maximization problem of a monopolist facing a market demand $\mu\left(T\left(p_{u}\right)\right)$ which is decreasing in $p_{u}$ :

$$
\max _{p_{u} \geq 0} u\left(T\left(p_{u}\right)\right) \cdot p_{u}
$$

We denote the seller's optimal (uniform) price and profit, obtained in the standard manner, as $p_{u}^{*}$ and $\pi_{u}^{*}$, respectively. Consequently, the consumer watches the movie in his favorite genre if his type $t$ satisfies $\bar{q} \cdot \max \left\{t_{A}, t_{B}\right\} \geq p_{u}^{*}$, and by the symmetry between the two movies the social welfare is calculated as

$$
W_{u}=\int_{T\left(p_{u}^{*}\right)} \bar{q} \cdot \max \left\{t_{A}, t_{B}\right\} d \mu=2 \int_{\max \left\{\frac{p_{u}^{*}}{q}, t_{B}\right\} \leq t_{A} \leq 1} \bar{q} \cdot t_{A} d \mu
$$

Thus, we have established and charaterized the equilibrium with the belief profile (3), which we call the "uniform price equilibrium." For expositional ease, we assume that the equilibrium is unique, which is indeed the case for generic distribution $\mu$.

For example, if $\mu$ is uniform on $[0,1]^{2}$ so that $\mu\left(T\left(p_{u}\right)\right)=1-\left(p_{u} / \bar{q}\right)^{2}$, the uniform price equilibrium values are calculated as

$$
p_{u}^{*}=\bar{q} / \sqrt{3}, \quad \pi_{u}^{*}=\bar{q} / \sqrt{3}-\bar{q}(3 \sqrt{3})=2 \bar{q} /(3 \sqrt{3}) \text { and } W_{u}=\frac{2 \bar{q}}{3}\left(1-\frac{1}{3 \sqrt{3}}\right)
$$

\section{Price Signalling Equilibrium}

We now characterize the equilibrium in which the consumer believes that the seller signals quality by pricing the high quality movie at a higher level, that is, the belief profile satisfies the following condition ${ }^{10}$ :

$$
\begin{cases}\beta_{A}(h \mid p)=\beta_{B}(\ell \mid p)=1 & \text { if } p_{A}>p_{B}, \\ \beta_{m}(h \mid p)=\beta_{m}(\ell \mid p)=0.5 \text { for } m \in\{A, B\} & \text { if } p_{A}=p_{B} \\ \beta_{A}(\ell \mid p)=\beta_{B}(h \mid p)=1 & \text { if } p_{A}<p_{B} .\end{cases}
$$

\footnotetext{
${ }^{10}$ For the case that $\varepsilon>0$, the probabilities 1 and 0.5 below would be $1-\varepsilon$ and $(1-\varepsilon) / 2$, respectively, in conjunction with $\beta_{A}(0 \mid p)=\beta_{B}(0 \mid p)=\varepsilon$.
} 
Given this belief, the seller obtains the same profit as in the uniform price equilibrium by setting $p_{A}=p_{B}=p_{u}^{*}$. We show below that she can do better by setting different prices for the two movies. To facilitate exposition, we normalize $\ell=1$ in the sequel.

Note that the two movies are symmetric in the sense that two price vectors, $\left(p_{A}, p_{B}\right)=\left(p^{\prime}, p^{\prime \prime}\right)$ and the "flipped" one $\left(p_{A}, p_{B}\right)=\left(p^{\prime \prime}, p^{\prime}\right)$ where $p^{\prime} \neq p^{\prime \prime}$, put the consumer in identical situations modulo relabeling of the movies, leading to identical decisions of the consumer (modulo relabeling of the movies) and the same level of profit for the seller. For expositional ease in characterizing the optimal prices given (6), therefore, without loss of generality we take the convention of labelling the high quality movie as $A$ in the sequel and focus on price vectors such that $p_{A}>p_{B}$.

Suppose that the seller sets $p_{A}>p_{B}$, signalling that $A$ is the good one. Then, the consumer watches a movie if and only if either $h t_{A} \geq p_{A}$ or $t_{B} \geq p_{B}$. More precisely, he watches $A$ if his type $t=\left(t_{A}, t_{B}\right)$ additionally satisfies

$$
h t_{A}-p_{A}>t_{B}-p_{B} \Leftrightarrow t_{A}>\frac{t_{B}+p_{A}-p_{B}}{h}
$$

and watches $B$ if the opposite inequality holds. It proves useful to illustrate such type-contingent decisions in the type space $T=[0,1]^{2}$ as below.

[Figure 1] Decision of consumer type $t=\left(t_{A}, t_{B}\right)$

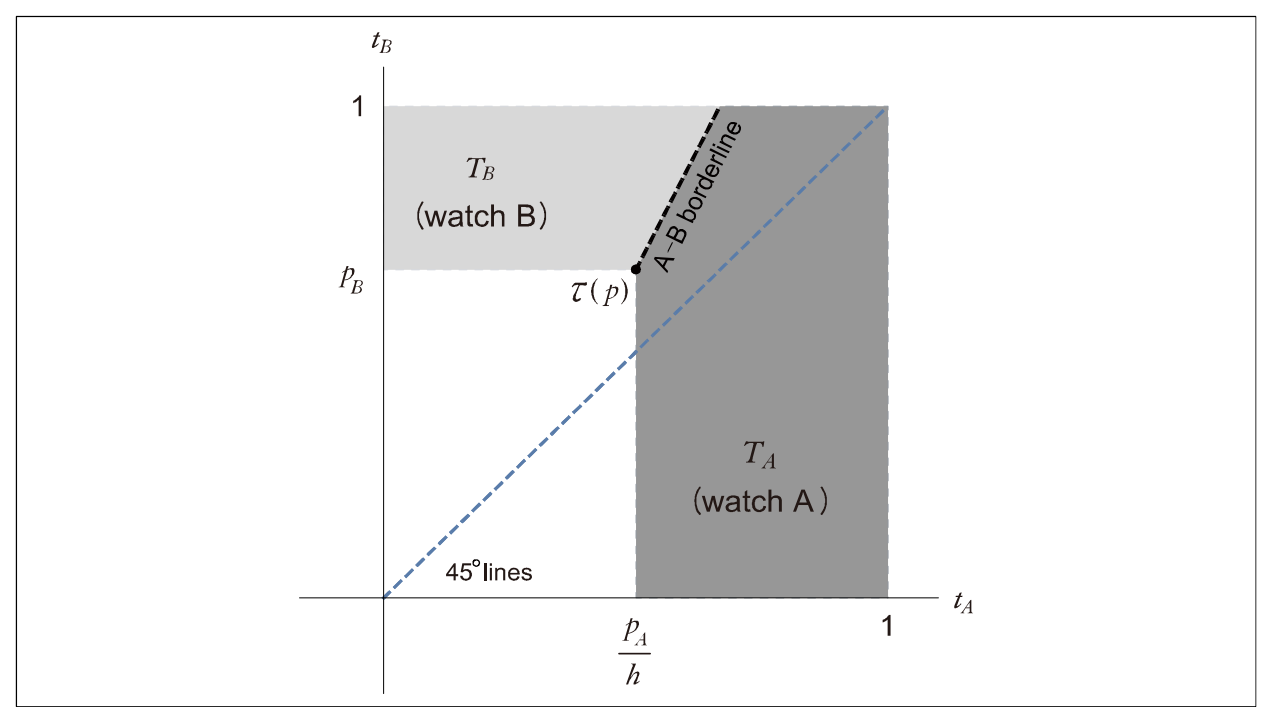

Given $p=\left(p_{A}, p_{B}\right)$ where $p_{A}>p_{B}$, let $\tau(p)=\left(\frac{p_{A}}{h}, p_{B}\right)$ denote the "critical type" who is indifferent among watching $A, B$, and neither, as depicted in Figure 
1. Moreover, the types $t \geq \tau(p)$ that satisfy (7) as an equality are depicted by a straight (dashed) line from $\tau(p)$ with a slope $h>1$ as illustrated in the diagram, which we call the " $A-B$ borderline." Then, given $p=\left(p_{A}, p_{B}\right)$, the consumer watches $A$ if his type $t=\left(t_{A}, t_{B}\right)$ is on the right hand side (RHS) of both the vertical line through $\tau(p)$ and the $A-B$ borderline, and watches $B$ if $t$ is above the horizontal line at the level $\tau(p)$ and on the left hand side (LHS) of the $A-B$ borderline.

Consequently, the seller solves

$$
\max _{0 \leq p_{B}<p_{A} \leq h} \pi\left(p_{A}, p_{B}\right):=p_{A} \mu\left(T_{A}\right)+p_{B} \mu\left(T_{B}\right)
$$

where $T_{A}=\left\{t \mid t_{A}>\max \left\{\frac{p_{A}}{h}, \frac{t_{B}+p_{A}-p_{B}}{h}\right\}\right\}$ and $T_{B}=\left\{t \mid t_{B}>\max \left\{p_{B}, h t_{A}-p_{A}+p_{B}\right\}\right\}$. The solution to $(8)$ is denoted by $\left(p_{A}^{*}, p_{B}^{*}\right)$ and called the "optimal signalling price." Then, together with the belief profile (6), the following constitutes a perfect Bayesian equilibrium (PBE): the seller sets $\left(p_{A}^{*}, p_{B}^{*}\right)$ and the consumer watches $A$ if $t \in T_{A}$, watches $B$ if $t \in T_{B}$, and neither otherwise. We refer to this equilibrium as the "price signalling equilibrium" and denote the seller's profit thereof by $\pi^{*}=\pi\left(p_{A}^{*}, p_{B}^{*}\right)$.

Remark 1. Given that $p_{B}=p_{A}$ is not in the constraint set, it is conceivable that (8) may not have a solution for some $\mu$ if the sup $\pi\left(p_{A}, p_{B}\right)$ is the limit value as $p_{B} \rightarrow p_{A}$. This is an inessential and technical issue of a continuous strategy set, stemming from the fact that real numbers are not well-ordered, and can be rectified by assuming (realistically) that there is a minimal monetary unit and the prices should be an integer multiple of it. We adopt this assumption whenever needed where the minimal unit is arbitrarily small, so that a solution to (8) is arbitrarily closely approximated by a solution when the constraint set is closed (which always exists by the Maximum Theorem). However, we believe that this issue rarely arises and does not arise for the class of distributions considered in Proposition 3 below, for instance. Moreover, the solution to (8) obtained as such generates a higher profit for the seller than the uniform price equilibrium, as shown below.

Remark 2. Other kinds of price signalling equilibria are also possible supported by belief profiles that differ from (6). In particular, if $\beta$ conforms to (6) for price vectors in a set $\tilde{P} \not\left(p_{A}^{*}, p_{B}^{*}\right)$ but conforms to the uniform price beliefs (3) for $p \in P \backslash \tilde{P}$, then the optimal price for the seller is the solution to (8) subject to $p \in \tilde{P}$ presuming that it is better than the uniform price vector $\left(p_{u}^{*}, p_{u}^{*}\right)$. Such equilibria require very specific coordination of consumer beliefs that treat some differential prices as signalling quality but others not, which seems unrealistic. 


\section{Stability, Profits and Welfare Comparison}

This section compares the two equilibria in several criteria. Specifically, we show that both equilibria are stable in the sense of Intuitive Criterion; the seller's profit is always higher in the price signalling equilibrium; but the consumer surplus and social welfare may be higher in either equilibrium depending on the distribution of consumer types.

\subsection{Stability}

Stability of equilibria in signalling games (broadly defined) is often questioned in terms of plausibility of off-equilibrium beliefs. We apply the Intuitive Criterion (Cho and Kreps, 1987), a well-known refinement concept for signalling games, and verify straightforwardly that both equilibria survive the criterion.

In our model, the seller (Sender) sends a "message" by setting a price pair $\left(p_{A}, p_{B}\right)$ based on her "type", i.e., private information on the realized qualities of the two movies. After an off-equilibrium message $\left(p_{A}, p_{B}\right)$ is sent, what the consumer does in the continuation game entirely determines the seller's payoff regardless of her type. Therefore, the maximum possible payoff of the seller from sending any off-equilibrium message $\left(p_{A}, p_{B}\right)$ is the same regardless of her type.

The seller's equilibrium payoff in the uniform price equilibrium, $\pi_{u}^{*}$, is also clearly the same regardless of her type. Hence, the seller of no type may be deemed inconceivable to have set any off-equilibrium price because she would at best do worse than following the equilibrium while she could have done better if she were of a different type (i.e., according to the Intuitive Criterion). Therefore, the uniform price equilibrium with the posterior belief (3) survives the Intuitive Criterion. The same argument and conclusion apply to the price signalling equilibrium as well, because the seller's payoff there, $\pi^{*}=\pi\left(p_{A}^{*}, p_{B}^{*}\right)$, is the same regardless of her private information as well (given the convention that we label the high quality movie as $A$ ).

\subsection{The Seller's Profit}

We now show that the seller can obtain higher profits with differential pricing than in the uniform price equilibrium. Recall that in the latter equilibrium the consumer watches the movie in his favorite genre if $\bar{q} \cdot \max \left\{t_{A}, t_{B}\right\} \geq p_{u}^{*}$ where $p_{u}^{*}$ is the optimal uniform price. This is depicted in Figure 2-(a), where the type $t_{u}^{*}=$ $\left(p_{u}^{*} / \bar{q}, p_{u}^{*} / \bar{q}\right)$ on the $45^{\circ}$ line is the critical type for whom watching $A$ or $B$ or neither are all equivalent. The seller's profit, therefore, is $p_{u}^{*}$ times the measure $\mu\left(T_{A} \cup T_{B}\right)=2 \mu\left(T_{A}\right)=2 \mu\left(T_{B}\right)$ where the latter two equalities are due to symmetry 
and $T_{A}$ and $T_{B}$ are as depicted in Figure 2-(a).

For comparison, suppose the seller signals quality by setting prices $\hat{p}_{A}=h p_{u}^{*} / \bar{q}$ and $\hat{p}_{B}=p_{u}^{*} / \bar{q}$, so that the critical type $\hat{t}:=\tau\left(\hat{p}_{A}, \hat{p}_{B}\right)$ coincides with the critical type $t_{u}^{*}$ above. Then, the same set of types watch a movie but some of them (those above the $45^{\circ}$ line and below the $A-B$ borderline) switch from watching $B$ to $A$ as in Figure 2-(b). Note that $\hat{p}_{A}-p_{u}^{*}=p_{u}^{*}(h-1) /(2 \bar{q})=p_{u}^{*}-\hat{p}_{B}>0$. Hence, relative to the uniform price equilibrium, (i) the seller collects the same total revenue from the types in $\hat{T}_{B}$ depicted in Figure 2-(b) and its mirror image along the $45^{\circ}$ line (a subset of $\left.\hat{T}_{A}\right)$ under $\left(\hat{p}_{A}, \hat{p}_{B}\right)$, and (ii) the remaining types in $\hat{T}_{A}$ (the kite-shape area symmetric around the $45^{\circ}$ line) pay $\hat{p}_{A}$ instead of $p_{u}^{*}$ under $\left(\hat{p}_{A}, \hat{p}_{B}\right)$. Since the seller's profit in the price signalling equilibrium is larger than that under $\left(\hat{p}_{A}, \hat{p}_{B}\right)$, we have established.

Proposition 1 The seller's profit is higher in the price signalling equilibrium than in the uniform price equilibrium.

[Figure 2] Decision under uniform pricing and differential pricing

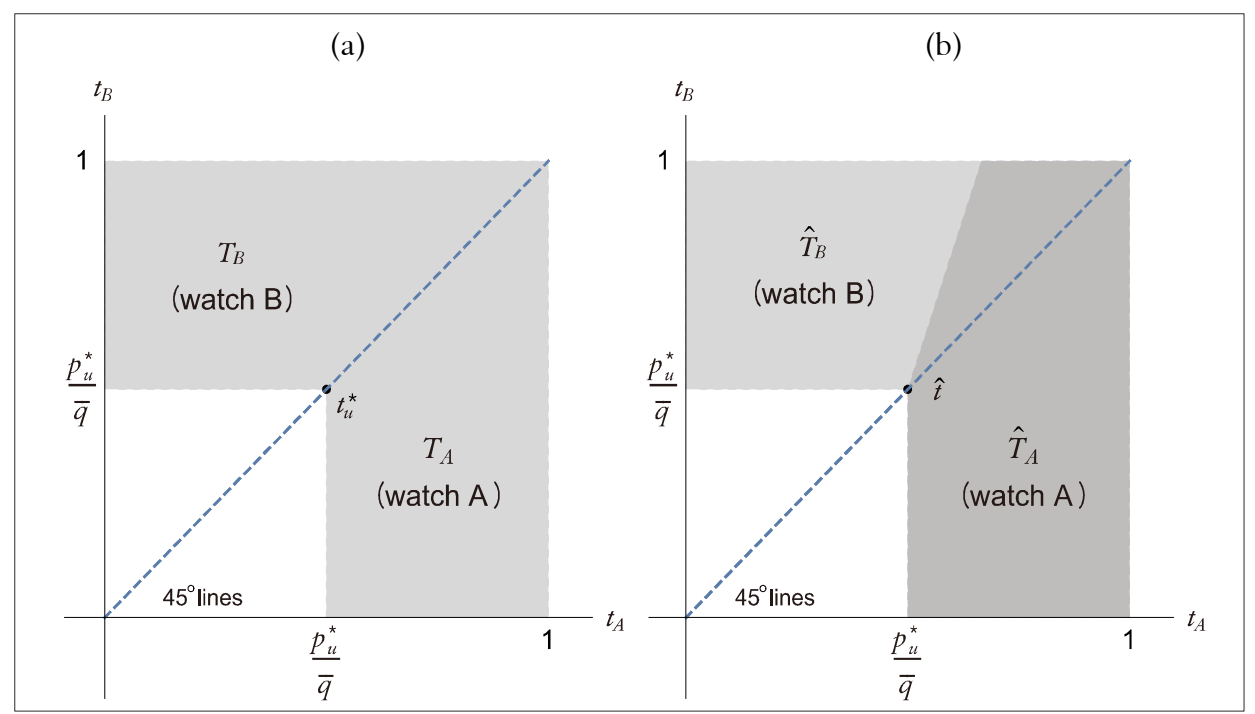

The key insight given above for this result is that the consumer is guided to choose the better quality movie for a higher price at the intensive margin, when the optimal uniform prices are differentiated to signal quality while keeping intact the set of types who would watch either movie. Hence, the seller is better off by price signalling in this way, and does even better by optimally exploiting the extensive margin as well. 


\subsection{Consumer Surplus and Social Welfare}

Note that the seller's profit gain in the price signalling equilibrium stems from more efficient choices of the consumer. The next question is whether the improved efficiency benefits the consumer as well, or is extracted to boost the seller's profit via higher prices. If the latter, does the boosted profit overshadow the reduction in consumer surplus so that the total welfare is higher with price signalling than without?

The answers to these questions will depend on how the extensive margin changes at the price signalling equilibrium relative to the uniform price equilibrium, which in turn is affected by the entire distribution of the consumer types. We show below that both consumer surplus and social welfare may be higher in either equilibrium.

We start with a sufficient condition for the social welfare and consumer surplus to be higher in the price signalling equilibrium. Recall the price vector $\left(\hat{p}_{A}, \hat{p}_{B}\right)=$ $\left(h p_{u}^{*} / \bar{q}, p_{u}^{*} / \bar{q}\right)$ for which the critical type $\hat{t}=\tau\left(\hat{p}_{A}, \hat{p}_{B}\right)$ coincides with the critical type $t_{u}^{*}=\left(p_{u}^{*} / \bar{q}, p_{u}^{*} / \bar{q}\right)$ in the uniform price equilibrium. Note that the welfare is higher under the signalling price vector $\left(\hat{p}_{A}, \hat{p}_{B}\right)$ than in the uniform price equilibrium, because the types who switch from watching $B$ to $A$ derive greater gross surpluses from movie $A$ (cf. Figure 2). Moreover, the consumer surplus is also higher as the increase in welfare exceeds the increase in the seller's profit (shown in Appendix).

It is intuitive that welfare is even higher with the optimal signalling price $\left(p_{A}^{*}, p_{B}^{*}\right)$ if the prices are lower for both movies than $\left(\hat{p}_{A}, \hat{p}_{B}\right)$. This is so if the price stays put for $A$ (i.e., $p_{A}^{*}=\hat{p}_{A}$ ) and is lowered for $B$ (i.e., $p_{B}^{*}<\hat{p}_{B}$ ) because (i) the seller's revenue increase from the types newly buying $B$ (which is less than the corresponding welfare increase) must overcompensate the revenue loss from the types switching from $A$ to $B$, and (ii) this revenue loss is larger than the corresponding decrease in welfare because the price differential, $p_{A}^{*}-p_{B}^{*}$, is the drop in consumption value from switching from $A$ to $B$ for types on the new AB borderline who experience the largest such drop from the switch. Then, the welfare must be higher if the price is lower for $A$ as well (i.e., $p_{A}^{*}<\hat{p}_{A}$ and $\left.p_{B}^{*}<\hat{p}_{B}\right)$.

This line of reasoning extends to the cases where the price is increased for $B$, but the price drop for $A$ is larger, that is, $\hat{p}_{A}-p_{A}^{*}>p_{B}^{*}-\hat{p}_{B}>0$. As the price changes from $\left(\hat{p}_{A}, \hat{p}_{B}\right)$ to $\left(p_{A}^{*}, p_{B}^{*}\right)$, a measure $\mu_{+A}=\mu\left\{t \mid \frac{p_{A}^{*}}{h}<t_{A}<\frac{\hat{p}_{A}}{h}, 0<t_{B}<\right.$ $\left.\hat{p}_{B}\right\}$ of types additionally buy movie $A$ while a measure $\mu_{-B}=\mu\left\{t \mid 0<t_{A}<\frac{p_{A}^{*}}{h}\right.$, $\left.\hat{p}_{B}<t_{B}<p_{B}^{*}\right\}$ of types cease to buy movie $B$. In addition, let $\mu_{A A}, \mu_{B B}$ and $\mu_{B A}$ denote the measure of types who stay with movie $A$, stay with movie $B$, and switch from $B$ to $A$, resp., as depicted in Figure 3. Because the revenue gain from the types in the sets $\mu_{+A} \cup \mu_{B A} \cup \mu_{B B}$ must exceed the loss from those in $\mu_{-B} \cup \mu_{A A}$, we deduce that the welfare gain for types in $\mu_{+A} \cup \mu_{B A}$ exceeds the 
welfare loss for types in $\mu_{-B}$. Since the welfare is already higher at $\left(\hat{p}_{A}, \hat{p}_{B}\right)$ than in the uniform price equilibrium, we conclude that price signalling enhances welfare. The same is shown to be the case for consumer surplus (in Appendix), establishing a sufficient condition stated below.

[Figure 3] Change of decisions by consumer types

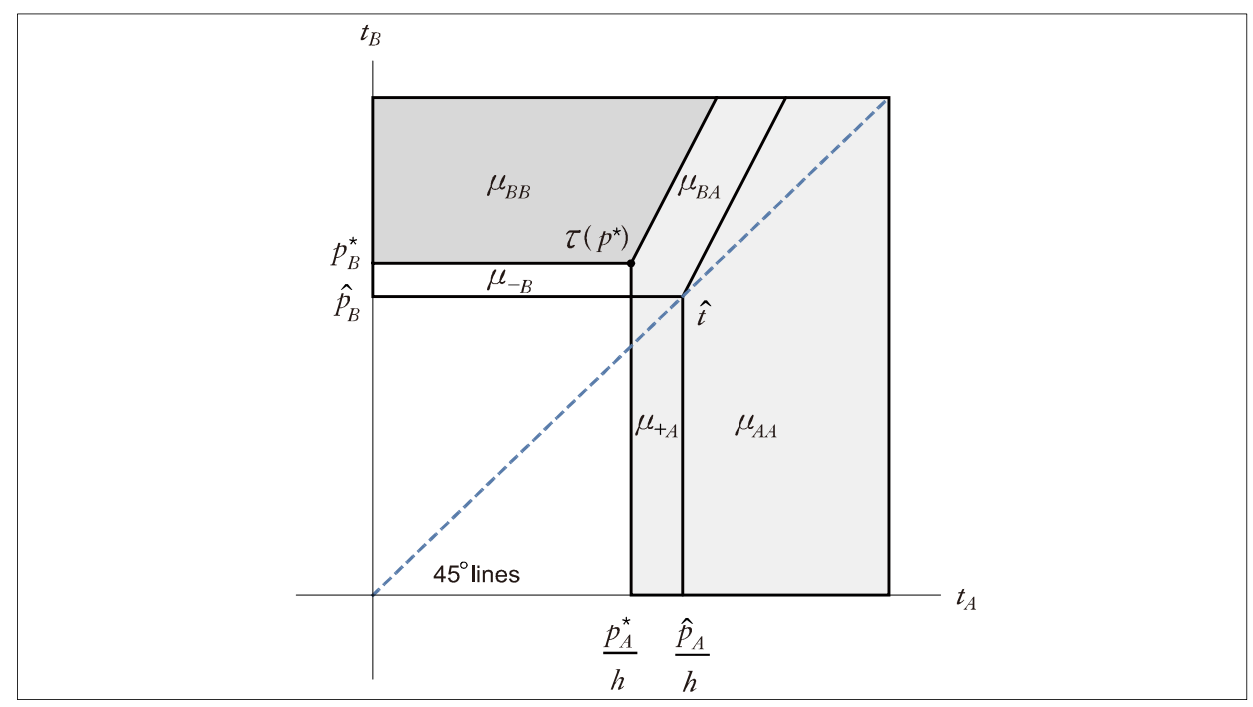

Proposition 2 The social welfare and consumer surplus are higher in the price signalling equilibrium $\left(p_{A}^{*}, p_{B}^{*}\right)$ than in the uniform price equilibrium $\left(p_{u}^{*}, p_{u}^{*}\right)$ if

$$
\hat{p}_{A}-p_{A}^{*}>\max \left\{p_{B}^{*}-\hat{p}_{B}, 0\right\}
$$

Proof. In Appendix A.

However, the sufficient condition (9) is not very useful as it is expressed in terms of solution values. It would be practically more useful to know when (9) holds in terms of the primitive data of the model, such as $\mu$, but a general characterization is difficult. Hence, we proceed our anaysis by focusing on the cases that the consumer's taste $t_{m}$ for each genre is independently distributed according to a common cdf $F(t)$ on $[0,1]$ with a continuous density function $f(t)=F^{\prime}(t)$.

We first derive a necessary condition which the optimal signalling price $\left(p_{A}^{*}, p_{B}^{*}\right)$ must satisfy in terms of the critical type $\tau\left(p_{A}^{*}, p_{B}^{*}\right)$ it induces, when the tastes are independent. Then, we identify a property implied by (9) on the derived necessary condition and thereby, discuss what kind of distributions $F$ may satisfy (9).

Consider a signalling price vector $p=\left(p_{A}, p_{B}\right)$ such that the maximal type $\bar{t}=$ $(1,1)$ is on the right side of the $A-B$ borderline. The seller's profit from $p$ 
given $F$ is

$$
\begin{aligned}
\pi(p)= & p_{A}\left[1-F\left(\frac{p_{A}}{h}\right)\right] F\left(p_{B}\right) \\
& +\int_{p_{B}}^{1}\left[p_{B} F\left(\frac{p_{A}+t_{B}-p_{B}}{h}\right)+p_{A}\left[1-F\left(\frac{p_{A}+t_{B}-p_{B}}{h}\right)\right]\right] d F\left(t_{B}\right) .
\end{aligned}
$$

Partially differentiating $\pi(p)$, we get

$$
\begin{aligned}
\frac{\partial \pi(p)}{\partial p_{A}}= & {\left[1-F\left(\frac{p_{A}}{h}\right)\right] F\left(p_{B}\right)-\frac{p_{A}}{h} f\left(\frac{p_{A}}{h}\right) F\left(p_{B}\right) } \\
& +\int_{p_{B}}^{1}\left[\frac{p_{B}-p_{A}}{h} f\left(\frac{p_{A}+t_{B}-p_{B}}{h}\right)+1-F\left(\frac{p_{A}+t_{B}-p_{B}}{h}\right)\right] d F\left(t_{B}\right), \\
\frac{\partial \pi(p)}{\partial p_{B}}= & -p_{B} F\left(\frac{p_{A}}{h}\right) f\left(p_{B}\right) \\
& +\int_{p_{B}}^{1}\left[F\left(\frac{p_{A}+t_{B}-p_{B}}{h}\right)+\frac{p_{A}-p_{B}}{h} f\left(\frac{p_{A}+t_{B}-p_{B}}{h}\right)\right] d F\left(t_{B}\right) .
\end{aligned}
$$

Note that $\frac{\partial \pi(p)}{\partial p_{B}}<0$ as $p_{B} \rightarrow 1$ so that $\pi(p)$ is not maximized at a price vector with $p_{B}=1$. Hence, a necessary condition for a solution $\left(p_{A}^{*}, p_{B}^{*}\right)$ to $(8)$ is ${ }^{11}$

$$
\begin{aligned}
& \frac{\partial \pi(p)}{\partial p_{A}}+\frac{\partial \pi(p)}{\partial p_{B}}=0 \Leftrightarrow \\
& F\left(\frac{p_{A}}{h}\right) F\left(p_{B}\right)+\frac{p_{A}}{h} f\left(\frac{p_{A}}{h}\right) F\left(p_{B}\right)+p_{B} F\left(\frac{p_{A}}{h}\right) f\left(p_{B}\right)=1 .
\end{aligned}
$$

In fact, (10) is also the analogous necessary condition calculated for price vectors such that the $A-B$ borderline has $\bar{t}=(1,1)$ on its left side (see Appendix B).

Similarly, since the profit from a uniform price $p_{u}$ is $p_{u}\left(1-F\left(\frac{p_{u}}{\bar{q}}\right)^{2}\right)$, a necessary condition for the uniform price equilibrium is

$$
1-F\left(\frac{p_{u}}{\bar{q}}\right)^{2}-2\left(\frac{p_{u}}{\bar{q}}\right) F\left(\frac{p_{u}}{\bar{q}}\right) f\left(\frac{p_{u}}{\bar{q}}\right)=0 \Leftrightarrow
$$

\footnotetext{
11 If the supremum of $\pi(p)$ is obtained somewhere on the boundary where $p_{A}=p_{B}$ (see Remark 1), say at $\left(p_{A}, p_{B}\right)=(\tilde{p}, \tilde{p})$, then $\pi(p)$ must be maximized at $(\tilde{p}, \tilde{p})$ along the said boundary. It is verified that the corresponding FOC implies that $(10)$ holds at $\left(p_{A}, p_{B}\right)=(\tilde{p}, \tilde{p})$.
} 


$$
\left[F\left(\frac{p_{u}^{*}}{\bar{q}}\right)\right]^{2}+2 \frac{p_{u}^{*}}{\bar{q}} F\left(\frac{p_{u}^{*}}{\bar{q}}\right) f\left(\frac{p_{u}^{*}}{\bar{q}}\right)=1
$$

Hence, condition $(10)$ holds at $p=\left(\hat{p}_{A}, \hat{p}_{B}\right)=\left(\frac{h p_{u}^{*}}{\bar{q}}, \frac{p_{u}^{*}}{\bar{q}}\right)$.

We now simplify the necessary condition (10). Recalling that $\left(\frac{p_{A}}{h}, p_{B}\right)=$ $\tau\left(p_{A}, p_{B}\right)$ is the critical type induced by a price vector $\left(p_{A}, p_{B}\right)$ under belief (6), we may rewrite $(10)$ as a function of critical type $t=\left(t_{A}, t_{B}\right)$ induced by price vector as follows (via a change of variables):

$$
Z(t):=F\left(t_{A}\right) F\left(t_{B}\right)+t_{A} f\left(t_{A}\right) F\left(t_{B}\right)+t_{B} F\left(t_{A}\right) f\left(t_{B}\right)=1 .
$$

This condition is symmetric between $t_{A}$ and $t_{B}$, hence facilitates exposition and discussion. Note that $Z(t)$ is nonnegative, assumes 0 when $t_{A}=0$ or $t_{B}=0$ and exceeds 1 when $t_{A}=t_{B}=1$.

Let us consider the cases that $Z(t)$ increases both in $t_{A}$ and in $t_{B}$. This is so if $t f(t)$ increases in $t$, for instance. Then, there is $t \in(0,1)$ such that $\left(10^{*}\right)$ holds at $\left(t_{A}, t_{B}\right)=(\underline{t}, 1)$ and $\left(t_{A}, t_{B}\right)=(1, \underline{t})$; moreover, there is a decreasing function

$$
\rho:[\underline{t}, 1] \rightarrow[\underline{t}, 1] \text { with } \rho(\underline{t})=1 \text { and } \rho(1)=\underline{t}
$$

that solves $\left(10^{*}\right)$ in the sense that $\left(10^{*}\right)$ holds at $\left(t_{A}, t_{B}\right)=\left(t_{A}, \rho\left(t_{A}\right)\right)$ for all $t_{A} \in$ $[\underline{t}, 1]$. Clearly, the graph of $\rho$ is symmetric around the $45^{\circ}$ line and crosses it at $t_{u}^{*}=\left(\frac{p_{u}^{*}}{\bar{q}}, \frac{p_{u}^{*}}{\bar{q}}\right)$, that is, $\rho\left(\frac{p_{u}^{*}}{\bar{q}}\right)=\frac{p_{u}^{*}}{\bar{q}}$ and has a slope of -1 at the crossing point, as illustrated in Figure 4. The critical type induced by the price signalling equilibrium, $\tau\left(p^{*}\right)=\left(\frac{p_{A}^{*}}{h}, p_{B}^{*}\right)$, is somewhere on the graph of $\rho$ by $(10)$.

Finally, we are ready to link the sufficient condition (9) with the properties of $\rho$ which is expressed in terms of the primitive data $F$ via $\left(10^{*}\right)$. Given the negative slope of $\rho$, the condition (9) implies that $\tau\left(p^{*}\right)$ is above the $45^{\circ}$ line but below the straight line going through the uniform price equilibrium critical type $t_{u}^{*}=\hat{t}$ with a slope $-h,{ }^{12}$ which we call "the line for (9)" and indicate by a dashed (red), negatively-sloped straight line from $t_{u}^{*}$ in Figure 4. In addition, the condition $p_{A}^{*}>p_{B}^{*}$ means that $\tau\left(p^{*}\right)$ is on the RHS of the straight line from the origin with a slope of $h$, called the "signalling price line" and indicated as another dashed (red) line in Figure 4. Therefore, if $\tau\left(p^{*}\right)$ is inside the triangle formed by these three lines, the welfare and consumer surplus are higher at the price signalling equilibrium by Proposition 2.

${ }^{12}$ That is, $\hat{p}_{A}-p_{A}^{*}>p_{B}^{*}-\hat{p}_{B}$ implies $p_{B}^{*}<\hat{p}_{B}-h\left(p_{A}^{*} / h-\hat{p}_{A} / h\right)$. 
[Figure 4] Condition for price signalling to improve welfare

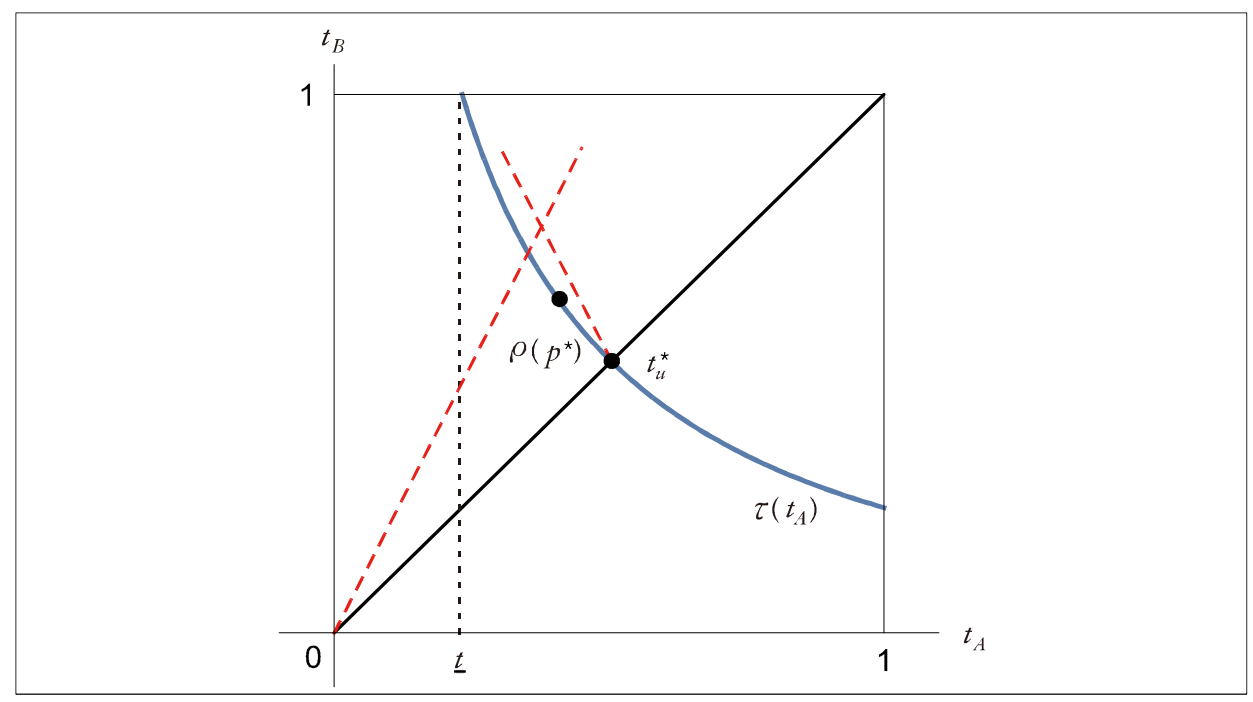

This condition is easily verified for the benchmark case of a uniform distribution $F(t)=t$. In this case, $\left(10^{*}\right)$ simplifies to $t_{A} t_{B}=1 / 3$ so that $\rho$ is convex, crosses the $45^{\circ}$ line at $t_{u}^{*}=\left(\frac{1}{\sqrt{3}}, \frac{1}{\sqrt{3}}\right)$, and has a slope $-\rho\left(t_{A}\right) / t_{A}$ which is $-h$ when $\rho\left(t_{A}\right)=h t_{A}$, i.e., when it crosses the signalling price line. Hence, $\tau\left(p^{*}\right)$ is inside the triangle formed by the three lines, provided that the profit $\pi(p)$ increases as the price vector changes so that the corresponding critical type moves northwest along the graph of $\rho$ below the $45^{\circ}$ line. This is indeed verified to be the case because $\frac{\partial \pi(p)}{\partial p_{B}}=\left(1+4 p_{A}-4 p_{B}-6 p_{A} p_{B}+3 p_{B}^{2}\right) /(2 h)$ is positive for $\left(p_{A}, p_{B}\right)$ such that $p_{B}<\frac{1}{\sqrt{3}}<\frac{p_{A}}{h}$. Consequently, price signalling improves social welfare and consumer surplus if $F$ is uniform.

By continuity, therefore, both the welfare and consumer surplus are higher in the price signalling equilibrium for all $F$ sufficiently close to a uniform distribution. This observation can be formalized in the following two manners:

(a) There is $r>0$ such that social welfare and consumer surplus are higher in the price signalling equilibrium if $|f(t)-1|<r$ for all $t \in[0,1]$.

(b) There is $r^{\prime}>0$ such that social welfare and consumer surplus are higher in the price signalling equilibrium if $\left|f^{\prime}(t)-1\right|<r^{\prime}$ for all $t \in[0,1]$.

To gain further insights, we focus on distributions with a linear density function, $f(t)=a+2(1-a) t$ for $a \in(0,2)$, and verify that price signalling enhances welfare for a large subset of such distributions in the next result. Recall that the function $\rho$ is well-defined if $t f(t)$ increases, which is the case for linear $f(t)$ if and only if 
$a \leq 4 / 3$.

Proposition 3 The social welfare and consumer surplus are higher in the price signalling equilibrium than in the uniform price equilibrium if $f(t)=a+2(1-a) t$ where $0<a$ $\leq 4 / 3$.

\section{Proof. In Appendix C.}

A general insight emerges from the discussions above: welfare is higher in the price signalling equilibrium when the density function $f(t)$ does not vary too wildly in $t$. Relative to the uniform price equilibrium, the seller increases sale of the high quality movie at the expense of low quality one by reducing qualityadjusted price for the former but raising it for the latter. The rise in the latter price is relatively small if $f(t)$ doesn't change rapidly because then the revenue loss from the raised price looms large given the already reduced volume of the low quality sale. Proposition 3 suggests that this result may prevail quite broadly. In fact, our Mathematica simulation for linear $f$ (presented in Figure 5) suggests that welfare is higher at the price signalling equilibrium for all $h$ if $a<1.8$.

[Figure 5] Comparison of social welfare

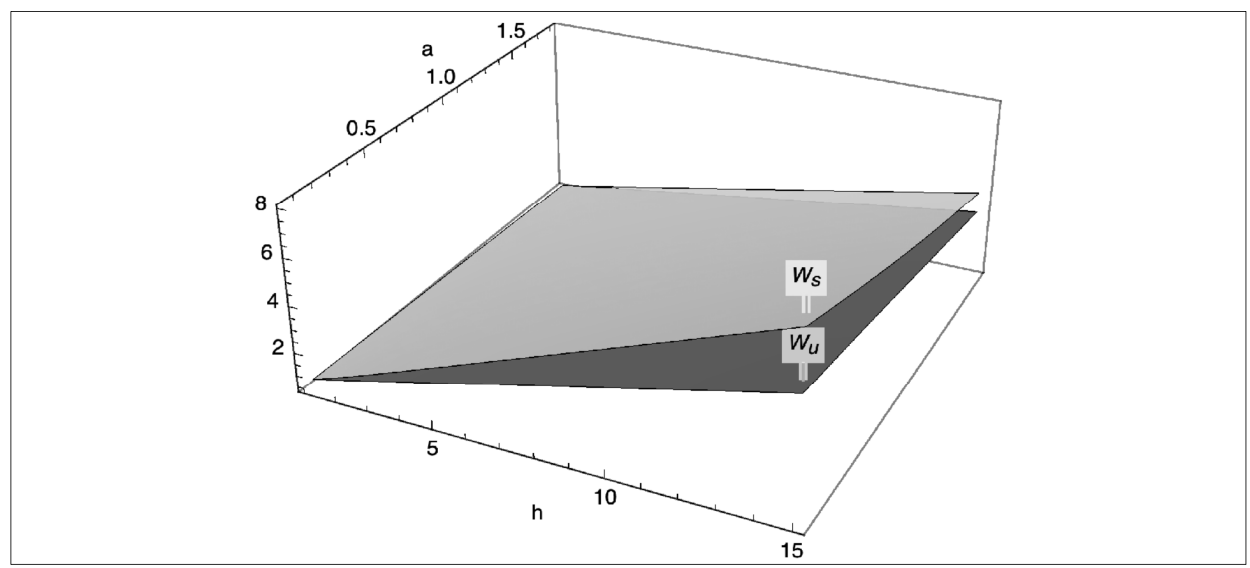

On the other hand, if the revenue loss from the raised price for the low quality movie is contained because $f(t)$ drops rapidly as $t$ increases from the uniform price critical type $t_{u}^{*}$, the seller may gain by raising it significantly to boost the revenue from the retained customers, resulting in a reduced welfare in the price signalling equilibrium. ${ }^{13}$ The size of the retained customers must be sufficiently

\footnotetext{
13 This is reminiscent of the third degree price discrimination arising from elasticity differences across consumer groups that can be separated. But, our model differs because the two market are inseparable and their demands are interrelated and endogenously determined by the prices set by the
} 
large for this to happnen, implying that $f(t)$ should be large enough for the types $t$ of the retained customers. This further means that $f(t)$ should surge after a sharp drop above the critical type $t_{u}^{*}$, implying a wild fluctuation of $f(t)$.

We provide a robust such example below. A general characterization of $F$ for which price signalling reduces welfare relative to the uniform price equilibrium, however, is a nontrivial task that is sensitive to various details of the function $F$ and goes beyond the scope of this paper.

Example: $F$ is concentrated on $t_{0}=0, t_{1}=0.5$ and $t_{2} \in(0.75,0.8)$.

Consider the case that $F$ has three equal point masses of $1 / 3$ each at $t_{0}, t_{1}$ and $t_{2}$ as above, so that the nine consumer types $t \in\left\{t_{0}, t_{1}, t_{2}\right\} \times\left\{t_{0}, t_{1}, t_{2}\right\}$ have an equal mass of $1 / 9$ each according to $\mu$. Suppose that $h$ is close to but less than $t_{2} / t_{1}$. We show that both the consumer surplus and social welfare are higher in the uniform price equilibrium in this case. Then, it is clear by continuity that the same conclusion prevails for all $F$ sufficiently nearby with a full support.

Uniform pricing regime : Consider prices $p_{u} \in[0, h]$ that the seller may set for both movies. If $p_{u} \leq t_{1} \bar{q}$ then all types except $t=(0,0)$ watch their favorate movie, generating a profit of $8 p_{u} / 9$ for the seller (assuming that the consumer watches a movie when he is indifferent between watching one and not at all). If $t_{1} \bar{q}<p_{u} \leq t_{2} \bar{q}$, all types with $t_{A}=t_{2}$ or $t_{B}=t_{2}$ watch their favorate movie, generating a profit of $5 p_{u} / 9$. If $p_{u}>t_{2} \bar{q}$, the profit is 0 . Since $8 t_{1} \bar{q} / 9=4 \bar{q} / 9>$ $5 t_{2} \bar{q} / 9$, the optimal uniform price is $p_{u}^{*}=t_{1} \bar{q}$. Thus, in the uniform price equilibrium, the seller's profit is $\pi_{u}^{*}=4 \bar{q} / 9$, the social welfare is $W_{u}=\bar{q}\left(3 t_{1}+\right.$ $\left.5 t_{2}\right) / 9$ assuming that the consumer watches one randomly when indifferent between the two movies, and the consumer surplus is $W_{u}-\pi_{u}^{*}=\bar{q}\left(3 t_{1}+5 t_{2}-4\right) / 9$.

Price signalling regime : It is clear that pricing to attract the lowest type $t=(0,0)$ is not worth it. The best differential pricing to attract all other types is to set $\left(p_{A}, p_{B}\right)=\left(h t_{1}, t_{1}\right),{ }^{14}$ which generates a profit of $\left(3 t_{1}+5 h t_{1}\right) / 9$ for the seller. She may increase $p_{B}$ arbitrarily close to $h t_{1}$ while keeping $p_{A}=h t_{1}$, increasing the revenue from types $\left(t_{0}, t_{2}\right)$ and $\left(t_{1}, t_{2}\right)$ by $h t_{1}-t_{1}$ at the cost of losing the revenue of $t_{1}$ from the type $\left(t_{0}, t_{1}\right)$. Since $2\left(h t_{1}-t_{1}\right) \approx 2\left(t_{2}-t_{1}\right)>0.5=t_{1}$ given $h \approx t_{2} / t_{1}$, the seller's profit is higher. As it is easily verified that she cannot increase her profit with any other prices, the seller's profit in the price signalling equilibrium is arbitrarily close to $\pi^{*}=7 h t_{1} / 9$, the social welfare to $W_{s}=\left(2 t_{2}+2 h t_{1}+3 h t_{2}\right) / 9$,

seller. For this reason, even when there is no elasticity difference in the absence of price signalling (so that the third degree price discrimination is irrelevant), differential pricing may enhance the social welfare as well as the profit (e.g., when $F$ is uniform).

${ }^{14}$ This is interpreted as the limit of $\left(p_{A}, p_{B}\right)=\left(h t_{1}-\varepsilon, t_{1}\right)$ as $\varepsilon \rightarrow 0$. 
and the consumer surplus to $W_{s}-\pi^{*}=\left(2 t_{2}-5 h t_{1}+3 h t_{2}\right) / 9$.

Therefore, the social welfare is higher in the uniform price equilibrium because

$$
W_{u}-W_{s}=\frac{3 t_{1}+t_{2}-\left(t_{1}+t_{2}\right) h}{18}>\frac{3 t_{1}+t_{2}-\left(t_{1}+t_{2}\right) t_{2} / t_{1}}{18}=\frac{3 t_{1}^{2}-t_{2}^{2}}{18 t_{1}}>\frac{11}{900}
$$

and so is the consumer surplus as $\bar{q}\left(3 t_{1}+5 t_{2}-4\right) / 9-\left(2 t_{2}-5 h t_{1}+3 h t_{2}\right) / 9=(2.5-$ $\left.t_{2}\right)(h-1) / 18>0$.

\section{Extension to Independent Movie Quality}

In this section we extend the results obtained in Sections 3-5 to the case that the two movie qualities $q_{m}$ for $m \in\{A, B\}$ are independent of each other. First, the off-equilibrium beliefs (3) continue to be valid in this case and the subsequent analysis is unaffected because it depends only on the expected quality; hence, the uniform price equilibrium is unaffected, either.

Next, we reformulate the price signalling equilibrium for the independent case. Recall that in Section 4 we have shown that

(*) if the consumer behaves optimally under the belief profile (6), i.e., believing that the expected quality of a movie is $h$ ( $\ell$, resp.) if it is priced higher (lower, resp.) than the other movie, then the seller maximizes her profit by pricing one movie at $p_{A}^{*}$ and the other at $p_{B}^{*}$ where $\left(p_{A}^{*}, p_{B}^{*}\right)$ solves $(8)$.

Hence, this pricing strategy constitutes an equilibrium together with the consumer's optimal decision given (6), so long as the expected quality of the movie priced at $p_{A}^{*}\left(p_{B}^{*}\right.$, resp. $)$ is indeed $h$ ( $\ell$, resp.). When the movie qualities are inversely correlated, this is the case (as shown in Section 4 ) if the seller prices

(a) the high quality movie at $p_{A}^{*}$ and the low quality movie at $p_{B}^{*}$.

In the current case where the two movie qualities are independent, suppose that the seller continues to price the two movies as described in (a) if they are of different qualities, but

(b) prices one movie at $p_{A}^{*}$ and the other at $p_{B}^{*}$ with equal probability if they are of the same quality. ${ }^{15}$

${ }^{15}$ Recall that we present analysis assuming $\varepsilon=0$. See Remark 3 below on how the analysis extends 
Then, by Bayes rule, a movie priced at $p_{A}^{*}$ is of high quality with probability $3 / 4$, whereas a movie priced at $p_{B}^{*}$ is of high quality with probability $1 / 4$.

Consequently, if we reinterpret $h$ and $\ell$ as the expected quality of a movie which is of high quality with probability $3 / 4$ and $1 / 4$, respectively, then the belief profile (6) is consistent with the seller's strategy of pricing as in (a) when the movie qualities are different and as in (b) otherwise. By (*) above, therefore, such pricing strategy of the seller constitutes, together with the consumer's optimal decision given (6), an equilibrium for the case that the movie qualities are independent. Note that the prices signal the quality less than perfectly in this case. Nevertheless, the discussions in Section 5 on stability, profits and welfare are not affected at all.

Remark 3. More generally, suppose that each $q_{m}$ is distributed according to a common distribution with a support $Q \subset \mathbb{R}_{+}$. Partition $Q$ into $Q_{h}$ and $Q_{\ell}$ so that the expected quality conditional on $q_{m} \in Q_{h}$ is $h$ and that conditional on $q_{m} \in Q_{\ell}$ is $\ell<h$. Then, the arguments above remain valid when all quality levels in $Q_{h}$ are treated identically (as "high" quality) and so are all levels in $Q_{\ell}$ (as "low" quality) in the seller's pricing strategy. This is so because the consumer's behavior is determined entirely by the expected qualities/beliefs associated with the prices, and the seller's profit is independent of her private information given such consumer's behavior.

\section{Conclusion}

We study equilibrium pricing behavior and its welfare implications in a stylized model of a single supplier of differentiated experience goods. Both uniform pricing and price signalling can prevail in equilibrium, supported by differently coordinated beliefs of consumers on the informational contents of the prices. This is in contrast with the general insight that price signalling is nonviable in static monopoly markets of a single experience good. The seller's profit is always higher in the price signalling equilibrium, but the welfare of the consumers and the society can be higher in the uniform pricing depending on market characteristics. Therefore, different pricing practices in different markets are consistent with equilibrium and possibly welfare-enhancing. We believe that these findings shed some new light on how the market operates for differentiated experience goods.

to the case that $q_{m}=0$ with probability $\varepsilon>0$. 


\section{Appendix}

\section{A. Proof of Proposition 2.}

It remains to consider the case that $p_{B}^{*}-\hat{p}_{B} \geq 0$. Recall the measures $\mu_{+A}$, $\mu_{-B}, \mu_{A A}, \mu_{B B}$ and $\mu_{B A}$ (cf. Figure 3). Since $\hat{p}_{A}-p_{A}^{*}>p_{B}^{*}-\hat{p}_{B} \geq 0$ by (9) and $\mu_{A A}>\mu_{B B}+\mu_{B A}+\mu_{-B}$, the seller's revenue from types who stay with the same movie decreases by $\mu_{A A}\left(\hat{p}_{A}-p_{A}^{*}\right)-\mu_{B B}\left(p_{B}^{*}-\hat{p}_{B}\right)>\left(\mu_{B B}+\mu_{B A}+\mu_{-B}\right)\left(\hat{p}_{A}-p_{A}^{*}\right)-$ $\mu_{B B}\left(p_{B}^{*}-\hat{p}_{B}\right)>\left(\mu_{B A}+\mu_{-B}\right)\left(\hat{p}_{A}-p_{A}^{*}\right)$.

For it to be optimal for the seller to set $\left(p_{A}^{*}-p_{B}^{*}\right)$, therefore, her revenue increase from $\mu_{+A}$ and $\mu_{B A}$ must exceed the loss from $\mu_{-B}$ by at least $\left(\mu_{B A}+\mu_{-B}\right)$ $\left(\hat{p}_{A}-p_{A}^{*}\right)$. The revenue increase from $\mu_{+A}$ is $\mu_{+A} p_{A}^{*}$ (lower than the consumer surplus increase), that from $\mu_{B A}$ is $\mu_{B A}\left(p_{A}^{*}-\hat{p}_{B}\right)$ whereas the corresponding increase in consumer surplus is at least $\mu_{B A}\left(p_{A}^{*}-p_{B}^{*}\right)$ because the types on the new $A-B$ borderline is willing to pay $p_{A}^{*}-p_{B}^{*}$ more for $A$ than for $B$, reflecting social surplus increase for such types. The revenue loss from $\mu_{-B}$ is $\mu_{-B} \hat{p}_{B}$ while the corresponding loss in social welfare is at most $\mu_{-B} p_{B}^{*}$. Consequently,

$$
\begin{aligned}
& \mu_{+A} p_{A}^{*}+\mu_{B A}\left(p_{A}^{*}-\hat{p}_{B}\right)-\mu_{-B} \hat{p}_{B} \geq\left(\mu_{B A}+\mu_{-B}\right)\left(\hat{p}_{A}-p_{A}^{*}\right) \\
\Rightarrow & \mu_{+A} p_{A}^{*}+\mu_{B A}\left(p_{A}^{*}-p_{B}^{*}\right)-\mu_{-B} p_{B}^{*} \geq\left(\mu_{B A}+\mu_{-B}\right)\left(\hat{p}_{A}-p_{A}^{*}+\hat{p}_{B}-p_{B}^{*}\right) \geq 0,
\end{aligned}
$$

i.e., social welfare is higher at the price signalling equilibrium than at $\left(\hat{p}_{A}, \hat{p}_{B}\right)$, which in turn is higher than at the uniform price equilibrium.

Moreover, as price vector changes from $\left(\hat{p}_{A}, \hat{p}_{B}\right)$ to $\left(p_{A}^{*}, p_{B}^{*}\right)$, the increase in social welfare is larger than the profit increase as calculated below:

$$
\begin{aligned}
& \mu_{+A} p_{A}^{*}+\mu_{B A}\left(p_{A}^{*}-p_{B}^{*}\right)-\mu_{-B} p_{B}^{*}-\left[\mu_{+A} p_{A}^{*}+\mu_{B A}\left(p_{A}^{*}-\hat{p}_{B}\right)-\mu_{-B} \hat{p}_{B}\right. \\
& \left.-\left(\mu_{B A}+\mu_{-B}\right)\left(\hat{p}_{A}-p_{A}^{*}\right)\right]=\left(\mu_{B A}+\mu_{-B}\right)\left(\hat{p}_{A}-p_{A}^{*}+\hat{p}_{B}-p_{B}^{*}\right)>0 .
\end{aligned}
$$

As the social welfare is the sum of consumer surplus and the seller's profit, this means that the consumer surplus increases as prices change from $\left(\hat{p}_{A}, \hat{p}_{B}\right)$ to $\left(p_{A}^{*}, p_{B}^{*}\right)$. Consequently, pricing signalling increases the consumer surplus provided that it is higher at the price vector $\left(\hat{p}_{A}, \hat{p}_{B}\right)$ than at the uniform price equilibrium, which we show to be the case below.

Recall from the discussion preceding Proposition 1 that, relative to the uniform price equilibrium, at $\left(\hat{p}_{A}, \hat{p}_{B}\right)$ the seller's profit increases by $\left(\hat{p}_{A}-p_{u}^{*}\right)$ times twice the measure of the types who switch from $B$ to $A$. For these types, movie $A$ is more valuable that $B$ by at least the price differential $p_{A}-p_{B}=2\left(\hat{p}_{A}-p_{u}^{*}\right)$ where the equality follows from $\hat{p}_{A}-p_{u}^{*}=p_{u}^{*}-\hat{p}_{B}$. Therefore, the welfare increases more than the seller's profit, implying that the consumer surplus is higher with the 
price vector $\left(\hat{p}_{A}, \hat{p}_{B}\right)$ than at the uniform price equilibrium, as desired.

B. Derivation of (10) for prices such that the $A-B$ borderline has $\bar{t}=(1,1)$ on its left side:

The seller's profit from such a signalling price $p=\left(p_{A}, p_{B}\right)$ is

$$
\pi(p)=p_{B}\left[1-F\left(p_{B}\right)\right] F\left(\frac{p_{A}}{h}\right)+\int_{p_{A} / h}^{1}\left[p_{B}+\left(p_{A}-p_{B}\right) F\left(p_{B}+h t_{A}-p_{A}\right)\right] d F\left(t_{A}\right) .
$$

Partially differentiating $\pi(p)$, we get

$$
\begin{aligned}
\frac{\partial \pi(p)}{\partial p_{A}}= & -\frac{p_{A}}{h} F\left(p_{B}\right) f\left(\frac{p_{A}}{h}\right) \\
& +\int_{p_{A} / h}^{1}\left[F\left(p_{B}+h t_{A}-p_{A}\right)-\left(p_{A}-p_{B}\right) f\left(p_{B}+h t_{A}-p_{A}\right)\right] d F\left(t_{A}\right) . \\
\frac{\partial \pi(p)}{\partial p_{B}}= & {\left[1-F\left(p_{B}\right)\right] F\left(\frac{p_{A}}{h}\right)-p_{B} f\left(p_{B}\right) F\left(\frac{p_{A}}{h}\right) } \\
& +\int_{p_{A} / h}^{1}\left[1-F\left(p_{B}+h t_{A}-p_{A}\right)+\left(p_{A}-p_{B}\right) f\left(p_{B}+h t_{A}-p_{A}\right)\right] d F\left(t_{A}\right),
\end{aligned}
$$

which establishes (10).

\section{Proof of Proposition 3.}

It is routinely verified that $\rho(\cdot)$ is strictly decreasing in $t_{A}$ from 1 at $t_{A}=\underline{t}$ where

$$
\underline{t}:=\frac{a^{2}-4 a+\sqrt{a^{4}-8 a^{3}+20 a^{2}-24 a+20}}{2\left(a^{2}-6 a+5\right)} \in\left[\frac{32-2 \sqrt{157}}{22}, \frac{1}{\sqrt{5}}\right],
$$

to $\rho(1)=\underline{t}$ at $t_{A}=1$. The value $\underline{t}$ decreases from $1 / \sqrt{5} \approx 0.4472$ from $a=0$ to $1 / 3$ at $a=1$ and to approximately 0.315 at $a=4 / 3$ as indicated above. There is a unique fixed point of $\rho$, which in fact is the critical type $t_{u}^{*}=p_{u}^{*} / \bar{q}$ in the uniform equilibrium price (with a slight abuse of notation $t_{u}^{*}$ ). Note that $t_{u}^{*}$ is independent of $\bar{q}$ (hence, of $h$ ) because $\bar{q}$ is just denotes the quality of the product and can be normalized in the uniform pricing regime.

Lemma a. $t_{u}^{*}$ has values in the interval $(0.525,0.67)$ and decreases in $a<4 / 3$. 
Proof. From $\left(10^{*}\right), t_{u}^{*}$ solves

$$
F(t)^{2}+2 t F(t) f(t)=t^{2}(a(1-t)+t)(5 t+a(3-5 t))=1 .
$$

It is routinely verified that $F(t)^{2}+2 t F(t) f(t)$ increases in $t$, has a value lower than 1 for all $a \in[0,1]$ when $t=0.525$, but has a value larger than 1 for all $a \in$ $[0,1]$ when $t=0.67$. Hence, $t_{u}^{*} \in(0.525,0.67)$. Since $F(t)^{2}+2 t F(t) f(t)$ increases both in $a$ and $t$ when $t \in(0.525,0.67), t_{u}^{*}$ decreases in $a \in(0,4 / 3)$.

Lemma b. $\rho:[\underline{t}, 1] \rightarrow[\underline{t}, 1]$ is convex.

Proof. Due to symmetry, it suffices to show convexity for $\rho:\left[t_{u}^{*}, 1\right] \rightarrow\left[\underline{t}, t_{u}^{*}\right]$. By differentiating both side of $\left.Z\right|_{t_{B}=\rho\left(t_{A}\right)}=0$ with respect to $t_{A}$ and rearranging, we get

$$
\rho^{\prime}\left(t_{A}\right)=-\left.\frac{F\left(t_{B}\right)\left[2 f\left(t_{A}\right)+t_{A} f^{\prime}\left(t_{A}\right)\right]+t_{B} f\left(t_{A}\right) f\left(t_{B}\right)}{F\left(t_{A}\right)\left[2 f\left(t_{B}\right)+t_{B} f^{\prime}\left(t_{B}\right)\right]+t_{A} f\left(t_{A}\right) f\left(t_{B}\right)}\right|_{t_{B}=\rho\left(t_{A}\right)}<0
$$

which is equal to the ratio of partial derivatives of $Z$ evaluated at $t_{B}=\rho\left(t_{A}\right)$, i.e.,

$$
\left.\frac{Z_{t_{A}}}{Z_{t_{B}}}\right|_{t_{B}=\rho\left(t_{A}\right)}=-\left.\frac{t_{B}\left[10 t_{A} t_{B}+4 a\left(2 t_{A}+t_{B}-5 t_{A} t_{B}\right)+a^{2}\left(3-4 t_{B}-8 t_{A}+10 t_{A} t_{B}\right)\right]}{t_{A}\left[10 t_{A} t_{B}+4 a\left(t_{A}+2 t_{B}-5 t_{A} t_{B}\right)+a^{2}\left(3-4 t_{A}-8 t_{B}+10 t_{A} t_{B}\right)\right]}\right|_{t_{B}=\rho\left(t_{A}\right)}
$$

We show below that the absolute value $\left|\frac{Z_{t_{A}}}{Z_{t_{B}}}\right|$ decreases in $t_{A}$ and increases in $t_{B}$. Given that $\rho^{\prime}\left(t_{A}\right)<0$, this implies that $\rho\left(t_{A}\right)$ is convex in $t_{A}$.

Differentiating $\left|\frac{Z_{t_{A}}}{Z_{t_{B}}}\right|$ with respect to $t_{A}$, we get

$$
\frac{-t_{B} \Psi_{A}}{t_{A}^{2}\left(10 t_{A} t_{B}+4 a\left(t_{A}+2 t_{B}-5 t_{A} t_{B}\right)+a^{2}\left(3-8 t_{B}+2 t_{A}\left(-2+5 t_{B}\right)\right)\right)^{2}}
$$

where

$$
\begin{aligned}
\Psi_{A}= & 100 t_{A}^{2} t_{B}^{2}+40 a t_{A} t_{B}\left(2 t_{B}+t_{A}\left(3-10 t_{B}\right)\right)+a^{4}\left(9-36 t_{B}+32 t_{B}^{2}-4 t_{A}\left(6-23 t_{B}+\right.\right. \\
& \left.\left.20 t_{B}^{2}\right)+4 t_{A}^{2}\left(8-30 t_{B}+25 t_{B}^{2}\right)\right)+4 a^{3}\left(t_{B}\left(9-16 t_{B}\right)+t_{A}\left(6-46 t_{B}+60 t_{B}^{2}\right)-\right. \\
& \left.2 t_{A}^{2}\left(8-45 t_{B}+50 t_{B}^{2}\right)\right)+4 a^{2}\left(t_{A}\left(23-60 t_{B}\right) t_{B}+8 t_{B}^{2}+2 t_{A}^{2}\left(4-45 t_{B}+75 t_{B}^{2}\right)\right) .
\end{aligned}
$$

Aiming to show $\Psi_{A}>0$, we calculate 


$$
\begin{aligned}
& \left.\Psi_{A}\right|_{t_{B}=0}=a^{2}\left(32 t_{A}^{2}(1-a)^{2}+a 24 t_{A}(1-a)+9 a^{2}\right)>0, \quad \forall 0<a<4 / 3 ; \\
& \frac{\partial \Psi_{A}}{\partial t_{B}}=4(1-a)\left[50 t_{A}^{2} t_{B}+a^{3}\left(9-t_{A}\left(23-40 t_{B}\right)-16 t_{B}+10 t_{A}^{2}\left(3-5 t_{B}\right)\right)+\right. \\
& \left.\quad 10 a t_{A}\left(4 t_{B}+3 t_{A}\left(1-5 t_{B}\right)\right)+a^{2}\left(16 t_{B}-30 t_{A}^{2}\left(2-5 t_{B}\right)+t_{A}\left(23-80 t_{B}\right)\right)\right] ;
\end{aligned}
$$

and

$$
\frac{\partial}{\partial t_{B}}\left(\frac{\partial \Psi_{A}}{\partial t_{B}}\right)=8(1-a)^{2}\left(25 t_{A}^{2}(1-a)^{2}+20 a t_{A}(1-a)+8 a^{2}\right)>0, \quad \forall 0<a<4 / 3
$$

Since $\left.\frac{\partial \Psi_{A}}{\partial t_{B}}\right|_{t_{B}=0}=4 a(1-a)\left(30 t_{A}^{2}(1-a)^{2}+23 a t_{A}(1-a)+9 a^{2}\right)>0$ for $a \in(0,1)$, therefore, it follows that $\Psi_{A}>0$ for $a \in(0,1)$. For $a \in(1,4 / 3)$, we calculate that $\left.\frac{\partial \Psi_{A}}{\partial t_{B}}\right|_{t_{B}=0}$ $<0$ and $\left.\frac{\partial \Psi_{A}}{\partial t_{B}}\right|_{t_{B}=1}<0$, as well as $\left.\Psi_{A}\right|_{t_{B}=1}>0$, verifying that $\Psi_{A}>0$. Consequently, $\left|\frac{Z_{t_{A}}}{Z_{t_{B}}}\right|$ decreases in $t_{A}$ as desired.

Next, differentiating $\left|\frac{Z_{t_{A}}}{Z_{t_{B}}}\right|$ with respect to $t_{B}$, we get

$$
\frac{\Psi_{B}}{t_{A}\left(10 t_{A} t_{B}+4 a\left(t_{A}+2 t_{B}-5 t_{A} t_{B}\right)+a^{2}\left(3-8 t_{B}+2 t_{A}\left(-2+5 t_{B}\right)\right)\right)^{2}}
$$

where

$$
\begin{aligned}
\Psi_{B}= & 100 t_{A}^{2} t_{B}^{2}+40 a t_{A} t_{B}\left(3 t_{B}+2 t_{A}\left(1-5 t_{B}\right)\right)+4 a^{3}\left(2 t_{B}\left(3-8 t_{B}\right)+t_{A}\left(9-46 t_{B}+\right.\right. \\
& \left.\left.90 t_{B}^{2}\right)+4 t_{A}^{2}\left(4-15 t_{B}+25 t_{B}^{2}\right)\right)+a^{4}\left(9-24 t_{B}+32 t_{B}^{2}+4 t_{B}^{2}\left(8-20 t_{B}+25 t_{B}^{2}\right)-\right. \\
& \left.4 t_{A}\left(9-23 t_{B}+30 t_{B}^{2}\right)\right)+4 a^{2}\left(t_{A}\left(23-90 t_{B}\right) t_{B}+8 t_{B}^{2}+2 t_{A}^{2}\left(4-30 t_{B}+75 t_{B}^{2}\right)\right) .
\end{aligned}
$$

Aiming to show that $\Psi_{B}>0$, we calculate

$$
\begin{aligned}
& \left.\Psi_{B}\right|_{t_{B}=0}=a^{2}\left(36 a t_{A}(1-a)+32 t_{A}^{2}(1-a)^{2}+9 a^{2}\right)>0 ; \\
& \frac{\partial \Psi_{B}}{\partial t_{B}}=4(1-a)\left(5 t_{A}(a-1)-2 a\right)\left(10 t_{A} t_{B}+4 a\left(t_{A}+2 t_{B}-5 t_{A} t_{B}\right)+a^{2}\left(3-8 t_{B}-\right.\right. \\
& \left.\left.\quad 2 t_{A}\left(2-5 t_{B}\right)\right)\right) ;
\end{aligned}
$$

and

$$
\frac{\partial}{\partial t_{B}}\left(\frac{\partial \Psi_{B}}{\partial t_{B}}\right)=8(1-a)^{2}\left(5 t_{A}(1-a)+4 a\right)\left(5 t_{A}(1-a)+2 a\right)>0
$$


Since $\left.\frac{\partial \Psi_{B}}{\partial t_{B}}\right|_{t_{B}=0}=4 a(1-a)\left(4 t_{A}(1-a)+3 a\right)\left(5 t_{A}(1-a)+2 a\right)>0$ for $a \in(0,1)$ therefore, it follows that $\Psi_{B}>0$ for $a \in(0,1)$. For $a \in(1,4 / 3)$, we calculate that $\left.\frac{\partial \Psi_{B}}{\partial t_{B}}\right|_{t_{B}=0}$ $<0$ and $\left.\frac{\partial \Psi_{B}}{\partial t_{B}}\right|_{t_{B}=1}<0$, as well as $\left.\Psi_{B}\right|_{t_{B}=1}>0$, verifying that $\Psi_{B}>0$. Consequently, $\left|\frac{Z_{t_{A}}}{Z_{t_{B}}}\right|$ increases in $t_{B}$ as desired.

Lemma c. If $\rho\left(t_{A}\right)<h t_{A}$ for $t_{A}<t_{u}^{*}$, then $\rho\left(t_{A}\right)<t_{u}^{*}+\left(t_{u}^{*}-t_{A}\right) h$.

Proof. Solving $F\left(t_{u}^{*}\right)^{2}+2 t_{u}^{*} F\left(t_{u}^{*}\right) f\left(t_{u}^{*}\right)=1$ for $a$, we get

$$
\alpha\left(t_{u}^{*}\right)=\frac{-4\left(t_{u}^{*}\right)^{3}+5\left(t_{u}^{*}\right)^{4}+\sqrt{\left(t_{u}^{*}\right)^{2}\left(3-8 t_{u}^{*}+5\left(t_{u}^{*}\right)^{2}+\left(t_{u}^{*}\right)^{4}\right)}}{\left(t_{u}^{*}\right)^{2}\left(3-8 t_{u}^{*}+5\left(t_{u}^{*}\right)^{2}\right)} .
$$

That is, $\alpha\left(t_{u}^{*}\right)$ is the value of $a \in(0,2)$ for which $t_{u}^{*}$ is the critical type in the uniform price equilibrium. For $t_{u} \in(0.525,0.67)$, therefore, it suffices to show for $a=\alpha\left(t_{u}\right)$ that $Z>1$ at $\left(t_{A}, t_{B}\right) \in\left[0, t_{u}\right] \times\left[t_{u}, 1\right]$ that solves $t_{B}=h t_{A}$ and $t_{B}=t_{u}$ $+\left(t_{u}-t_{A}\right) h$ if exists, or that solves $t_{B}=1=t_{u}+\left(t_{u}-t_{A}\right) h<h t_{A}$.

The former solution is $t_{A}=(1+h) t_{u} /(2 h)$ and $t_{B}=(1+h) t_{u} / 2$ so long as $(1+h) t_{u} / 2<1$, i.e., $h<\left(2 / t_{u}\right)-1$. The value of $Z$ evaluated at that solution for $a=\alpha\left(t_{u}\right)$ is

$$
\begin{aligned}
& \left(\frac{(1+h) t_{u}}{4 h}\right)^{2} \cdot\left[5(1+h)^{2} t_{u}^{2}-\frac{2(1+h)^{2}\left(4-5 t_{u}\right)\left(4 t_{u}^{2}-5 t_{u}^{3}-\sqrt{3-8 t_{u}+5 t_{u}^{2}+t_{u}^{4}}\right)}{3-8 t_{u}+5 t_{u}^{2}}\right. \\
& \left.+\frac{\left(t_{u}\left(8-5 t_{u}\right)\left(1+h^{2}\right)-2 h\left(6-8 t_{u}+5 t_{u}^{2}\right)\right)\left(4 t_{u}^{2}-5 t_{u}^{3}-\sqrt{3-8 t_{u}+5 t_{u}^{2}+t_{u}^{4}}\right)^{2}}{t_{u}^{2}\left(3-8 t_{u}+5 t_{u}^{2}\right)^{2}}\right]
\end{aligned}
$$

which is routinely calculated to be 1 when $h=1$. The partial derivative of the expression in the big bracket with respect to $h$ is

$$
\begin{aligned}
& 10(1+h) t_{u}^{2}+\frac{4(1+h)\left(5 t_{u}-4\right)\left(4 t_{u}^{2}-5 t_{u}^{3}-\sqrt{3-8 t_{u}+5 t_{u}^{2}+t_{u}^{4}}\right)}{3-8 t_{u}+5 t_{u}^{2}} \\
& +\frac{2\left(6+t_{u}\left(5 t_{u}-8\right)(1+h)\right)\left(4 t_{u}^{2}-5 t_{u}^{3}-\sqrt{3-8 t_{u}+5 t_{u}^{2}+t_{u}^{4}}\right)^{2}}{t_{u}^{2}\left(3-8 t_{u}+5 t_{u}^{2}\right)^{2}}
\end{aligned}
$$

which is linear in $h$ with values $4 / t_{u}^{2}$ at $h=1$ and 


$$
4 \times \frac{15+51 t_{u}^{3}-120 t_{u}^{4}+75 t_{u}^{5}-40 t_{u}+25 t_{u}^{2}+\left(30 t_{u}^{2}-24 t_{u}\right) \sqrt{3-8 t_{u}+5 t_{u}^{2}+t_{u}^{4}}}{\left(t_{u}-1\right) t_{u}^{2}\left(3-5 t_{u}\right)^{2}}
$$

at $h=\left(2 / t_{u}\right)-1$, both of which are positive for $t_{u} \in(0.525,0.67)$. Since $(1+h) / h$ also increases in $h$, therefore, the value of $Z$ evaluated at $t_{A}=(1+h) t_{u} /(2 h)$ and $t_{B}=(1+h) t_{u} / 2$ for $a=\alpha\left(t_{u}\right)$, (12), exceeds 1 so long as $h<\left(2 / t_{u}\right)-1$, as desired. For $h>\left(2 / t_{u}\right)-1$, since $Z$ increases in $t_{A}$, it follows that $Z$ exceeds 1 at $t_{B}=1$ and $t_{A}=\left(t_{u}(1+h)-1\right) / h$, as desired.

Proposition 2 applies as the condition (9) holds by Lemmas b and c so long as the price signalling equilibrium $\left(p_{A}^{*}, p_{B}^{*}\right)$ satisfies $p_{A}^{*} / h<p_{B}^{*}$. This is indeed the case by the next lemma. (Below we return to use $p_{A}$ rather than $t_{A}=p_{A} / h$ in calculations.)

Lemma d. $\frac{\partial \pi(p)}{\partial p_{B}}>0$ along $\rho$ if $p_{B}<t_{A}=p_{A} / h$.

Proof. Recall $t_{u}^{*}$ decreases in $a$ from 0.66874 at $a=0$ to 0.63116 at $a=1 / 2$, to 0.6 at $a=22 / 27 \approx 0.815$, to 0.577 at $a=1$, and to 0.525 at $a=4 / 3$.

(i) We show that $\frac{\partial \pi(p)}{\partial p_{B}} \geq 0$ at $p=\left(h t_{u}^{*}, t_{u}^{*}\right)$. From $\frac{\partial \pi(p)}{\partial p_{B}}$ evaluated at $p_{A}=h p_{B}$, we get $X /\left(6 h^{2}\right)$ where

$$
\begin{aligned}
X= & 3+16(h-1) p_{B}+18(h-1)^{2} p_{B}^{2}-5\left(1-4 h+6 h^{2}\right) p_{B}^{4}+a^{2}\left(1-p_{B}\right)\left(1-3 p_{B}-\right. \\
& \left.3 p_{B}^{2}+5 p_{B}^{3}-6 h^{2} p_{B}^{2}\left(3-5 p_{B}\right)-h\left(1-3 p_{B}-12 p_{B}^{2}+20 p_{B}^{3}\right)\right)+2 a((1- \\
& \left.p_{B}\right)^{2}\left(6 p_{B}+5 p_{B}^{2}-2\right)+3 h^{2} p_{B}\left(2-3 p_{B}-8 p_{B}^{2}+10 p_{B}^{3}\right)+2 h\left(1-8 p_{B}+\right. \\
& \left.\left.9 p_{B}^{2}+8 p_{B}^{3}-10 p_{B}^{4}\right)\right) .
\end{aligned}
$$

We need to show that $X>0$ for all relevant values of $p_{B}=t_{u}^{*}$.

To do this, we first observe that conditional on $h=1, \frac{\partial \pi(p)}{\partial p_{B}}=\frac{\partial \pi(p)}{\partial p_{A}}$ when evaluated at $p_{A}=p_{B}$ and must be both 0 at $p_{A}=p_{B}=p_{u}^{*}=t_{u}^{*}$ because otherwise, e.g., if $\frac{\partial \pi(p)}{\partial p_{B}}=\frac{\partial \pi(p)}{\partial p_{A}}>0$, then the seller would increase her profit by increasing the common price above $p_{u}^{*}$, violating optimality of $p_{u}^{*}$. Hence, $\left.X\right|_{h=1, p_{B}=p_{u}^{*}}=0$.

Therefore, it suffices to show that

$$
\begin{aligned}
\partial X / \partial h= & 4 p_{B}\left(4+9(-1+h) p_{B}+(5-15 h) p_{B}^{3}\right)-a^{2}\left(-1+p_{B}\right)\left(-1+3 p_{B}+(12-\right. \\
& \left.36 h) p_{B}^{2}+20(-1+3 h) p_{B}^{3}\right)+4 a\left(1+(-8+6 h) p_{B}-9(-1+h) p_{B}^{2}+\right. \\
& \left.(8-24 h) p_{B}^{2}+10(-1+3 h) p_{B}^{4}\right)
\end{aligned}
$$

is positive for all $h \geq 1$ at $p_{B}=t_{u}^{*}$ and $a=\alpha\left(t_{u}^{*}\right)$ for relevant values of $t_{u}^{*} \in$ 
$(0.52,0.67)$. We do this by showing that

$$
\begin{aligned}
\partial X /\left.\partial h\right|_{h=1}= & 8 p_{B}\left(2-5 p_{B}^{3}\right)+a^{2}\left(-1+4 p_{B}-27 p_{B}^{2}+64 p_{B}^{3}-40 p_{B}^{4}\right)+a\left(4-8 p_{B}-\right. \\
& \left.64 p_{B}^{3}+80 p_{B}^{A}\right)>0
\end{aligned}
$$

and also that

$$
\begin{gathered}
\partial^{2} X / \partial h^{2}=-12 p_{B}\left(p_{B}\left(-3-5 p_{B}^{2}\right)+a^{2} p_{B}\left(3-8 p_{B}+5 p_{B}^{2}\right)+a\left(-2+3 p_{B}+8 p_{B}^{2}-\right.\right. \\
\left.\left.10 p_{B}^{3}\right)\right)>0
\end{gathered}
$$

for relevant values. Specifically, $\partial X /\left.\partial h\right|_{h=1}$ is (i) concave in a when the coefficient of $a^{2}$ is negative (which is the case if $p_{B}<0.546 \ldots$ ) peaking at a value of $a<0$, and achieves a positive value at $a=4 / 3$, and (ii) convex in $a$ when the coefficient of $a^{2}$ is positive (which is the case if $p_{B}>0.546 \ldots$ ) bottoming out at a value of $a>4 / 3$, and achieves a positive value at $a=\alpha\left(p_{B}\right)$. Hence, $\partial X /\left.\partial h\right|_{h=1}>0$ is verified. Similarly, $\partial^{2} X / \partial h^{2}$ is (i) convex in $a$ when the coefficient of $a^{2}$ is positive (which is the case if $p_{B}<0.6$ ) bottoming out at a value of $a<0$, and achieves a positive value at $a=0$, and (ii) concave in $a$ when the coefficient of $a^{2}$ is negative (which is the case if $p_{B}>0.6$ ) peaking at a value of $a>4 / 3$, and achieves a positive value at $a=\alpha\left(p_{B}\right)$. Hence, $\partial^{2} X / \partial h^{2}>0$ is verified.

(ii) We show that $\frac{\partial \pi(p)}{\partial p_{B}}$ decreases in $p_{B}$ and increases in $p_{A}$ for $\left(p_{A}, p_{B}\right) \in$ $\left[h p_{B}, h\right] \times\left[0, t_{u}^{*}\right]$. (The calculation below is when $A-B$ borderline hits top line. The other case is analogous.) To show the latter, we calculate that $\frac{\partial}{\partial p_{A}}\left[\frac{\partial \pi(p)}{\partial p_{B}}\right]=Y /\left(3 h^{2}\right)$ where

$$
\begin{aligned}
Y:= & 2\left(4-9 p_{B}+5 p_{B}^{3}+p_{A}\left(9-15 p_{B}^{2}\right)\right)+2 a\left(9 p_{B}+6 p_{B}^{2}-10 p_{B}^{3}+h\left(3-6 p_{B}^{2}\right)-\right. \\
& \left.3 p_{A}\left(33_{4}+p_{B}-10 p_{B}^{2}\right)-5\right)+a^{2}\left(3 h p_{B}\left(4 p_{B}-3\right)+2\left(1-6 p_{B}^{2}+5 p_{B}^{3}+\right.\right. \\
& \left.\left.3 p_{A} p_{B}\left(4-5 p_{B}\right)\right)\right) .
\end{aligned}
$$

Verifying that $\frac{\partial \pi(p)}{\partial p_{B}}$ increases in $p_{A}$ amounts to showing that $Y>0$ for relevant ranges of variable values. Note that $\partial Y / \partial p_{A}=6(1-a)\left(3-5 p_{B}^{2}+a p_{B}(-4+\right.$ $\left.\left.5 p_{B}\right)\right)$ is positive (neg.) for $a<1 \quad(a>1)$. For $\mathrm{a}<1$, therefore, it suffices to show that $Y$ is positive at $p_{A}=h p_{B}$. From

$$
\begin{aligned}
\left.Y\right|_{p_{A}=h p_{B}}= & 2\left(4+9(-1+h) p_{B}+(5-15 h) p_{B}^{3}\right)+a^{2}\left(-3 h p_{B}\left(3-12 p_{B}+10 p_{B}^{2}\right)+\right. \\
& \left.2\left(1-6 p_{B}^{2}+5 p_{B}^{3}\right)\right)+2 a\left(-5+9 p_{B}+6 p_{B}^{2}-10 p_{B}^{3}+3 h\left(1-3 p_{B}-\right.\right. \\
& \left.\left.6 p_{B}^{2}+10 p_{B}^{3}\right)\right)
\end{aligned}
$$


we calculate that $\frac{\left.\partial Y\right|_{p_{A}=h p_{B}}}{\partial h}=6 p_{B}\left(3-5 p_{B}^{2}\right)-3 a^{2} p_{B}\left(3-12 p_{B}+10 p_{B}^{2}\right)+6 a\left(1-3 p_{B}-6 p_{B}^{2}\right.$ $\left.+10 p_{B}^{3}\right)$ and that $\left.Y\right|_{p_{A}=h p_{B}, h=1}=8-20 p_{B}^{3}+a^{2}\left(2-9 p_{B}+24 p_{B}^{2}-20 p_{B}^{3}\right)+4 a\left(-1-6 p_{B}^{2}\right.$ $\left.+10 p_{B}^{3}\right)$, both of which are routinely verified to be positive for $a<\alpha\left(p_{B}\right)$ and $p_{B}<0.67,{ }^{16}$ establishing that $Y$ is positive for $a<1$.

Similarly, for $a \in(1,4 / 3)$ it suffices to show that $Y$ is positive at $p_{A}=h$. From

$$
\begin{aligned}
\left.Y\right|_{p_{A}=h}= & 2\left(4-9 p_{B}+5 p_{B}^{3}+h\left(9-15 p_{B}^{2}\right)\right)+2 a\left(-5+9 p_{B}+6 p_{B}^{2}+10 p_{B}^{3}+6 h(-1-\right. \\
& \left.\left.2 p_{B}+4 p_{B}^{2}\right)\right)+a^{2}\left(-3 h p_{B}\left(-5+6 p_{B}\right)+2\left(1-6 p_{B}^{2}+5 p_{B}^{3}\right)\right)
\end{aligned}
$$

we calculate that $\frac{\left.\partial Y\right|_{p_{A}=h}}{\partial h}=18-30 p_{B}^{2}-3 a^{2} p_{B}\left(-5+6 p_{B}\right)+12 a\left(-1-2 p_{B}+4 p_{B}^{2}\right)$ and that $\left.Y\right|_{p_{A}=h, h=1}=2\left(13-9 p_{B}-15 p_{B}^{2}+5 p_{B}^{3}\right)-2 a\left(11+3 p_{B}-30 p_{B}^{2}+10 p_{B}^{3}\right)+a^{2}\left(2+15 p_{B}\right.$ $\left.-30 p_{B}^{2}+10 p_{B}^{3}\right)$, both of which are routinely verified to be positive for $p_{B}<1 / 3$.

Since $\rho\left(t_{A}\right)$ is convex with values of $\rho\left(t_{u}^{*}\right)=t_{u}^{*}$ and $\rho(1)=\underline{t}$, for $p_{B} \in(1 / 3$, $0.57)$ we only need to show $Y$ to be positive at $p_{A}=\left(0.57+\left(0.57-p_{B}\right) \frac{1-0.57}{0.57-1 / 3}\right) h$. This can be shown similarly: evaluate $Y$ at this value of $p_{A}$, and verify that both its derivative with respect to $h$ and its value at $h=1$ are positive for $p_{B} \in(1 / 3$, 0.57). This establishes that $\frac{\partial \pi(p)}{\partial p_{B}}$ increases in $p_{A}$.

To show that $\frac{\partial \pi(p)}{\partial p_{B}}$ decreases in $p_{B}$, observe that the integrand (the expression inside the square bracket) in $\frac{\partial \pi(p)}{\partial p_{B}}$ is fully determined by $p_{A}-p_{B}$, hence decreasing $p_{B}$ has the same effect as increasing $p_{A}$. Since decreasing $p_{B}$ additionally expands the range of integration, enlarging the effect on the integral value. The effect on the first term of $\frac{\partial \pi(p)}{\partial p_{B}}$ is positive from decreasing $p_{B}$ but negative from increasing $p_{A}$. Since $\frac{\partial \pi(p)}{\partial p_{B}}$ increases in $p_{A}$ as shown above, it follows that it increases as $p_{B}$ decreases, as desired.

This completes the proof of Lemma $\mathrm{d}$ and, thus, the proof of Proposition 3.

\footnotetext{
${ }^{16}$ Specifically, they are shown to be positive for $p_{B}<0.67$ if $a<0.5$ and for $p_{B}<0.64$ if $a \in(0.5,1)$.
} 


\section{References}

Cho, I.-K. and D. M. Kreps (1987), “Signalling Game and Stable Equilbria," Quarterly Journal of Economics, 102, 179-221.

Heidhues, P. and B. Koszegi (2008), "Competition and Price Variation When Consumers Are Loss Averse," American Economic Review, 98, 1245-1268.

Judd, K. and M. H. Riordan (1994), "Price and Quality in a New Product Monopoly," Review of Economic Studies, 61, 773-789.

Mas-Colell, A., M. D. Whinston and J. Green (1995), Microeconomic Theory, Oxford Uni-versity Press, New York.

Milgrom, P. and J. Roberts (1986), "Price and Advertising Signals of Product Quality," Journal of Political Economy, 94, 796-821.

Miller, J., R. Stone and E. Stuen (2013), "Evidence of Convergence to a Signalling Equilib-rium for Califorenia Wine 2001-2005," Journal of Research in Industrial Organization, Article ID 443296, 12 pages.

Nelson, P. (1970), “Information and Consumer Behavior,” Journal of Political Economy, 78, 311-329.

Orbach, B. Y. and L. Einav (2007), "Uniform Prices for Differentiated Goods: The Case of the Movie-theater Industry," International Review of Law and Economics, 27, 129-153.

Richardson, M. and F. Stahler (2016), "On the "Uniform Pricing Puzzle" in Recorded Music," Information Economics and Policy, 34, 58-66.

Wolinsky, A. (1983), "Prices as Signals of Product Quality,” Review of Economic Studies, 50, 647-658. 